\title{
Neural Activity Selects Myosin IIB and VI with a Specific Time Window in Distinct Dynamin Isoform-Mediated Synaptic Vesicle Reuse Pathways
}

\author{
Michikata Hayashida, ${ }^{1}$ Shota Tanifuji, ${ }^{1}$ Huan Ma, ${ }^{1}$ Noriko Murakami, ${ }^{2}$ and $\mathbb{C}^{-S u m i k o ~ M o c h i d a ~}{ }^{1}$ \\ ${ }^{1}$ Department of Physiology, Tokyo Medical University, Tokyo 160-8402, Japan and ${ }^{2}$ Department of Molecular Biology, New York State Institute for Basic \\ Research in Developmental Disabilities, New York 10314
}

\begin{abstract}
Presynaptic nerve terminals must maintain stable neurotransmissions via synaptic vesicle (SV) resupply despite encountering wide fluctuations in the number and frequency of incoming action potentials (APs). However, the molecular mechanism linking variation in neural activity to SV resupply is unknown. Myosins II and VI are actin-based cytoskeletal motors that drive dendritic actin dynamics and membrane transport, respectively, at brain synapses. Here we combined genetic knockdown or molecular dysfunction and direct physiological measurement of fast synaptic transmission from paired rat superior cervical ganglion neurons in culture to show that myosins IIB and VI work individually in SV reuse pathways, having distinct dependency and time constants with physiological AP frequency. Myosin VI resupplied the readily releasable pool (RRP) with slow kinetics independently of firing rates but acted quickly within 50 ms after AP. Under high-frequency AP firing, myosin IIB resupplied the RRP with fast kinetics in a slower time window of 200 ms. Knockdown of both myosin and dynamin isoforms by mixed siRNA microinjection revealed that myosin IIB-mediated SV resupply follows amphiphysin/dynamin-1-mediated endocytosis, while myosin VI-mediated SV resupply follows dynamin-3-mediated endocytosis. Collectively, our findings show how distinct myosin isoforms work as vesicle motors in appropriate SV reuse pathways associated with specific firing patterns.
\end{abstract}

Key words: action potential; dynamin; endocytosis; myosin; synaptic vesicle

\section{Introduction}

The actin cytoskeleton in brain synapses (Korobova and Svitkina, 2010) regulates synaptic function and plasticity as well as synapse morphology (Cingolani and Goda, 2008; Frost et al., 2010; Hotulainen and Hoogenraad, 2010; Kneussel and Wagner, 2013). Myosins are a large family of actin-based cytoskeletal motors that use energy derived from ATP hydrolysis to generate movement and force (Kneussel and Wagner, 2013). The class II (Vicente-Manzanares et al., 2009), V (Hammer and Sellers, 2012), and VI myosins (Sweeney and Houdusse, 2010) have specific roles required for synapse function (Osterweil et al., 2005; Wagner et al., 2011; Peng et al., 2012) and several forms of synaptic plasticity (Yano et al., 2006; Rex et al., 2010). Their major roles at synapses are diverse and include the regulation of actin cytoskeleton dynamics in dendritic spines and powering of synaptic cargo transport (Wagner et al., 2011).

\footnotetext{
Received Dec. 10, 2014; revised May 2, 2015; accepted May 6, 2015.

Author contributions: S.M. designed research; M.H., S.T., H.M., and S.M. performed research; N.M. contributed unpublished reagents/analytic tools; M.H., S.T., and S.M. analyzed data; S.M. wrote the paper.

This work was supported by grants-in-aid for Scientific Research B for Exploratory Research \#25290025 (S.M.). The authors declare no competing financial interests.

Correspondence should be addressed to Sumiko Mochida, Department of Physiology, Tokyo Medical University, Tokyo 160-8402, Japan. E-mail: mochida@tokyo-med.ac.jp.

H. Ma's present address: Department of Physiology and Neuroscience, New York University, New York 10016. DOI:10.1523/JNEUROSCI.5028-14.2015

Copyright $\odot 2015$ the authors $\quad 0270-6474 / 15 / 358901-13 \$ 15.00 / 0$
}

We first demonstrated the role of myosin in regulation of neurotransmitter release at presynaptic terminals (Mochida et al., 1994a), where myosin IIB is highly expressed (Takagishi et al., 2005). Recent live imaging of hippocampal neurons provided direct evidence that myosin II is required for synaptic vesicle (SV) motility during synaptic firing (Peng et al., 2012). Importance of myosin II in SV motility and synaptic transmission was also demonstrated at Drosophila melanogaster neuromuscular junction (Seabrooke and Stewart, 2011; Seabrooke et al., 2010). There, myosin VI is needed for SV localization, short-term plasticity (Kisiel et al., 2011), and SV dynamics (Kisiel et al., 2014). In contrast, in brain synapses, myosin VI is postsynaptically involved in AMPAR (Osterweil et al., 2005; Nash et al., 2010) and GABA receptor trafficking (Kneussel and Wagner, 2013). Its role in SV motility at presynaptic terminals is not yet explored.

At presynaptic terminals, clusters of SVs are called SV pools. SVs in the readily releasable pool (RRP) undergo exocytosis upon $\mathrm{Ca}^{2+}$ influx. The RRP is thought to be replenished from a larger SV cluster called a recycling pool, which maintains transmitter release under physiological synaptic activity (Rizzoli and Betz, 2005). How to mobilize SV from one cluster to another is not yet understood. At the calyx of Held synapses, myosin II controls the RRP (Lee et al., 2010). During a prolonged depolarizing stimulation, the RRP can be divided into a fast and a slowly releasing pool (Neher and Sakaba, 2008). In response to high-frequency firing myosin II converts slowly releasing SVs to fast-releasing ones (Lee 
et al., 2010), suggesting that myosin II motors are important for maintaining release-ready SVs (RRSVs) during higher levels of synaptic activity.

Presynaptic terminals must supply RRSVs into the RRP to maintain stable neurotransmission via SV recycling despite encountering wide fluctuations in the number and frequency of incoming action potentials (APs). Here we combined presynaptic genetic knockdown or molecular dysfunction and direct physiological measurement of synaptic transmission from the paired SCG neurons. We discovered that presynaptic myosin IIB and VI work individually in distinct vesicle reuse pathways activated by dynamin isoforms, having distinct rate and time constants with physiological AP frequency.

\section{Materials and Methods}

Ethical approval

The Ethics Committee of Tokyo Medical University approved this project.

\section{Materials}

siRNAs and antibodies were all purchased from Santa Cruz Biotechnology; the catalog numbers of siRNAs for myosin IIA, IIB, VI, dynamin1,dynamin-3, and control negative were sc-61121, sc-61123, sc-37134, sc-35234, sc-41209, and sc-36869, respectively, whereas those of antibodies for myosin IIA, IIB, or VI were sc-47199, sc-47205, or sc-50461, respectively.

\section{Neuronal culture and transfection}

P7 Wistar ST rats of either sex were decapitated under diethyl ether anesthesia according to the Guidelines of the Physiological Society of Japan. Isolated and mixed SCG neurons were cultured for 5-7 weeks and microinjected (Ma and Mochida, 2007; Tanifuji et al., 2013) with myosin IIA, IIB, VI, dynamin-1, dynamin-3, or control-negative siRNA. To monitor the injected amount and to detect injected neurons, siRNA was introduced along with EGFP-DNA (a gift from Dr. Sakisaka, Kobe University, Japan). The reduction in myosin and dynamin expression was confirmed by a decrease in fluorescence intensity of immunocytochemical staining (Baba et al., 2005; Krapivinsky et al., 2006; Tanifuji et al., 2013) 36-42 h after microinjection.

\section{Analysis of immunostaining images}

SCG neurons 36-42 h after myosin IIB, VI, or control-siRNA injection were stained as described previously (Tanifuji et al., 2013). Anti-myosin IIB or VI (sc-47205 or sc-50461; 1:50 dilution) was used for the primary antibody. Alexa Fluor 546 goat anti-mouse IgG or Alexa Fluor 546 goat anti-rabbit IgG (A-11003 or A-11035; Invitrogen; 1:500) was used for the secondary antibody. Confocal images were obtained with a Nikon EZ-C2 microscope using a water-immersion $60 \times$ objective. Images of myosin IIB and VI in the siRNA-injected neurons and an adjacent noninjected neuron were acquired using the same settings below saturation level at a resolution of $1024 \times 1024$ pixels (12-bit). The density of myosin IIB and VI was quantified by averaging fluorescence intensity within five circles (10 $\mu^{2}$ each) on the EGFP-expressing neuronal processes in the siRNA-injected cells and an adjacent noninjected cell. The regions of interest for comparison of fluorescence intensity between siRNAinjected and noninjected neurons in an image that contains two to three dots of myosin IIB or VI were randomly chosen and background fluorescence intensity in a $10 \mu \mathrm{m}^{2}$ circle was subtracted.

\section{EPSP recordings}

Presynaptic neurons $2 \mathrm{~d}$ or $3 \mathrm{~d}$ after siRNA injection (Krapivinsky et al., 2006) or immediately after anti-myosin IIA, IIB, or VI antibody injection (Mochida et al., 1994a) or IIB heavy chain fragment injection (Takagishi et al., 2005) were used. Neurons were superfused with a modified Krebs' solution consisting of $136 \mathrm{~mm} \mathrm{NaCl}, 5.9 \mathrm{mM} \mathrm{KCl}, 2.5 \mathrm{mM} \mathrm{CaCl}_{2}, 1.2 \mathrm{mM}$ $\mathrm{MgCl}_{2}, 11$ mm glucose, and $3 \mathrm{~mm}$ Na-HEPES, pH 7.4 (32-34 ${ }^{\circ} \mathrm{C}$; Lu et al., 2009; Ma et al., 2009). Presynaptic APs were generated by passing current through a sharp recording electrode filled with $1 \mathrm{M} \mathrm{K}$-acetate (70-90
$\mathrm{M} \Omega$ ), and EPSPs were recorded from a neighboring nontransfected neuron. Data were collected using Clampex10.2 (Molecular Devices).

\section{Intracellular injection procedure}

From the start of EPSP recording in response to consecutive lowfrequency AP firing $(0.05-0.2 \mathrm{~Hz})$, the amplitude decreases gradually for 10-20 min and then stabilizes (Mochida, 1995; Mochida et al., 1994a). Thus, after initial decrease in EPSP and following 20-30 min of stable EPSP recording, each myosin antibody or heavy chain fragment of myosin IIA or IIB was injected for 3-4 min into the presynaptic cell body by diffusion from a patch pipette (15-20 M $\Omega$ tip resistance). Fast Green FCF (1\%; Sigma-Aldrich) was applied with the agents to visualize their entry into the presynaptic cell body through the disrupted membrane and to estimate their amounts in the cell body. Reagents to be injected were dissolved in a solution consisting of $150 \mathrm{~mm}$ potassium acetate, $5 \mathrm{~mm}$ Mg-ATP, and 10 mM HEPES, pH 7.4.

Analysis of EPSPs with various AP firing patterns

Data were analyzed with Origin software (OriginLab).

EPSPs with low-frequency trains. To measure reduction of releaseready SVs (RRSVs) in response to consecutive low-frequency AP firing, an AP was elicited in a presynaptic neuron at $0.05,0.1$, and $0.25 \mathrm{~Hz}$. EPSP amplitudes were normalized to the mean EPSP amplitude from $20 \mathrm{~min}$ recording before antibody or myosin fragment injection or the mean value from the first three EPSP amplitudes. Normalized and averaged EPSP amplitudes were smoothed using Origin 85 points adjacent averaging. To compare the rate of RRSV reduction, the normalized values were fitted with exponential curves using Origin 7.5 fit exponential decay with first order or second order.

EPSPs with high-frequency trains and the RRP size. To measure reduction of RRSVs in response to high-frequency AP firing, $2 \mathrm{~s}$ AP trains at 5, 10 , and $20 \mathrm{~Hz}$ were consecutively elicited. For each frequency, AP trains were performed in triplicate every $2 \mathrm{~min}$. To compare changes in SVs in the RRP during $2 \mathrm{~s}$ AP trains, the peak EPSP amplitude measured from the baseline was normalized to the first EPSP amplitude. The RRP size was estimated from back-extrapolation (to time $=0$ ) of average cumulative EPSP amplitudes recorded at 10 and $20 \mathrm{~Hz}$ (Schneggenburger et al., 1999) using Origin 8. The number of synaptic vesicles in the RRP was estimated by dividing the value of the RRP size with the mean value of the quantal EPSP amplitude (0.4 mV; Krapivinsky et al., 2006).

Paired-pulse ratio. To examine RRSVs following AP generation, two consecutive APs at various interstimulus intervals (ISIs) were elicited, every $1 \mathrm{~min}$, in the siRNA or myosin heavy chain fragment-injected neurons. Recordings for each ISI were performed in triplicate. To calculate the paired-pulse ratio, the second EPSP amplitude measured from the end of the first EPSP was normalized by the amplitude of the first EPSP measured from the baseline before generating the first EPSP. The mean values of the paired-pulse ratio for individual synapse were calculated.

$R R P$ depletion and recovery. EPSP was recorded at $1 \mathrm{~Hz}$. After a $1 \mathrm{~min}$ control recording at $1 \mathrm{~Hz}$, a 3 min or 4 min stimulation at $5 \mathrm{~Hz}$ was applied to deplete SVs in the RRP. EPSP amplitudes were normalized to the mean EPSP amplitude before the AP trains. Averaged EPSP amplitudes were smoothed using Origin 8 five points adjacent averaging.

Time constant for RRP recovery. Averaged EPSP amplitudes were fitted with single exponential growth curves by $15 \mathrm{~s}$, and with double exponential growth curves by 8 min after the AP train, using Origin 7.5 fit exponential growth. To show clearly the fast recovery rate, the mean value of noise level of the baseline recording at time $=0(<1 \mathrm{mV})$ was subtracted from the mean EPSP amplitudes. To show clearly the slow recovery rate, the EPSP amplitude was normalized to the mean value of the EPSP amplitude from 0.51 to $0.6 \mathrm{~min}$ and fitted with a single exponential curve.

Statistics. Statistical significance was determined by the two-tailed Student's $t$ test or one-way ANOVA. All data are shown as the mean with SEM. Statistical significance was assumed when $p<0.05, p<0.03, p<$ 0.01 , or $p<0.001$. 

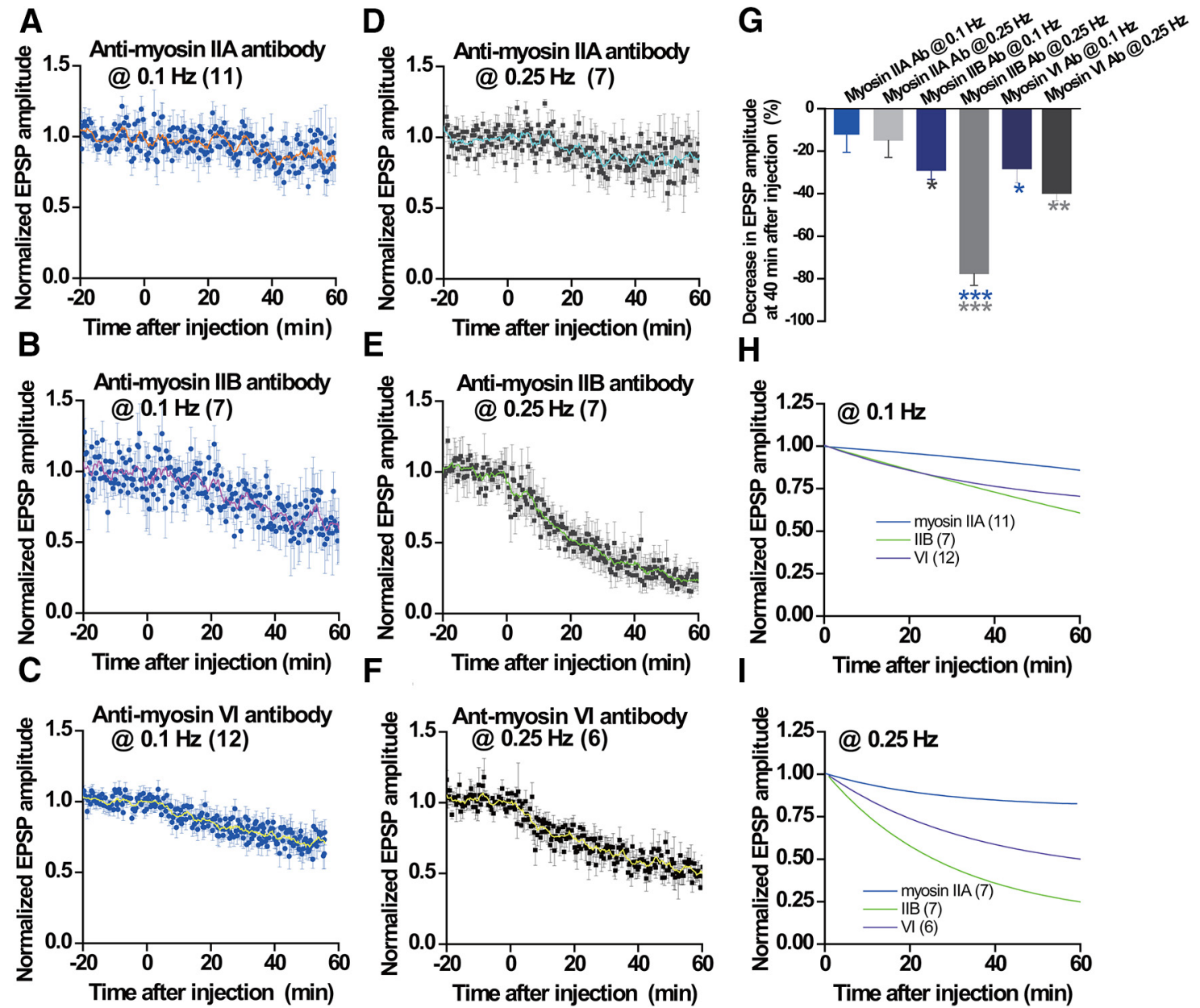

Figure 1. Myosin IIB or VI dysfunction reduces RRSVs during consecutive transmitter release. At $t=0$ presynaptic neurons were injected with antibody against myosin IIA, IIB, or VI. EPSP amplitude recorded at $0.1 \mathrm{~Hz}$ or $0.25 \mathrm{~Hz}$ was normalized to the mean EPSP amplitude from a 20 min recording before antibody injection $(n=6-12)$. $A-F$, Averaged EPSP amplitudes (circle and square) with SEM (bar) and the smoothed values with a 5-point moving averaged algorithm (line). G, Decrease in EPSP amplitudes at 40 min after the start of antibody injection. ${ }^{* * *} p<0.001$, ${ }^{* *} p<0.01,{ }^{*} p<0.05$; Bonferroni post hoc test after one-way ANOVA, $p<0.001$ : IIB at $0.25 \mathrm{~Hz}$ vs myosin IIA (blue, $0.1 \mathrm{~Hz} ;$ gray, $0.25 \mathrm{~Hz}$ ), $p<0.01, \mathrm{Vl}$ at $0.25 \mathrm{~Hz}$ vs IIA at $0.25 \mathrm{~Hz}, p<0.05$, IIB at $0.1 \mathrm{~Hz}$ vs IIB at $0.25 \mathrm{~Hz}$ and VI at $0.1 \mathrm{~Hz}$ vs IIB at $0.25 \mathrm{~Hz} ; p>0.05$, VI at $0.1 \mathrm{~Hz}$ vs $0.25 \mathrm{~Hz}$. $\boldsymbol{H}, \boldsymbol{I}$, Averaged EPSP amplitudes in $\boldsymbol{A}-\boldsymbol{F}$ were fitted with single exponential decay curves. Bonferroni post hoc test after one-way ANOVA, $p<0.001 \| A$ at $0.1 \mathrm{~Hz}$ vs $\| B$ at $0.1 \mathrm{~Hz}$, IIA at $0.1 \mathrm{~Hz}$ vs VI at $0.1 \mathrm{~Hz}, \| \mathrm{A}$ at $0.25 \mathrm{~Hz}$ vs $\| \mathrm{B}$ at $0.25 \mathrm{~Hz}$, IIA at $0.25 \mathrm{~Hz}$ vs VI at $0.25 \mathrm{~Hz}, \| \mathrm{A}$ at $0.25 \mathrm{~Hz}$ v V V at $0.25 \mathrm{~Hz} ; p<$ 0.05 , IIB at $0.1 \mathrm{~Hz}$ vs VI at $0.1 \mathrm{~Hz}$, IIB at $0.1 \mathrm{~Hz}$ vs $0.25 \mathrm{~Hz} ; p>0.05$, VI at $0.1 \mathrm{~Hz}$ vs $0.25 \mathrm{~Hz}$.

\section{Results}

Myosins IIB and VI participate in regulation of RRSVs

We have shown that presynaptic myosin II dysfunction by an antibody against the actin binding site reduced synaptic activityinduced transmitter release from SCG neurons (Mochida et al., 1994a) and that IIB, but not IIA, Va, or Vb, is specifically expressed at the presynaptic terminals (Takagishi et al., 2005). Here we first confirmed myosin IIB participation in the RRP resupply by monitoring the recovering kinetics of RRSVs in presynaptic terminals dialyzed with isoform-specific antibodies during lowfrequency synaptic activity consisting of consecutive presynaptic APs at $0.1 \mathrm{~Hz}$ or $0.25 \mathrm{~Hz}$ (Fig. $1 A-F)$. Consistent with the previous studies for myosin II (Mochida et al., 1994a), anti-IIB antibody injected into a presynaptic neuron gradually decreased the EPSP over the $50 \mathrm{~min}$ recording (Fig. $1 B, E$ ). In contrast, anti-IIA antibody as a control antibody dialysis (Fig. $1 A, D$ ) showed timedependent decrease similarly seen with control IgG dialysis (Mochida et al., 1994a). The reduction in EPSP amplitude at 40 min after the injection shows that the IIB antibody effect is significant (Fig. $1 G$ ). The time course for RRSV reduction can be estimated by fitting the mean EPSP amplitudes with an exponen- tial decay curve (Fig. $1 H, I$ ). Dysfunction of IIB showed a more rapid RRSV reduction than that of IIA levels of reduction following repetitive APs at both firing rates (Fig. $1 H, I$ ). In addition, the decay time course for IIB dysfunction was significantly faster at $0.25 \mathrm{~Hz}$ than at $0.1 \mathrm{~Hz}$, suggesting an activity-dependent action for myosin IIB.

When myosin IIB action was perturbed by injecting the recombinant heavy chain fragments (IIB-wt, $47 \mathrm{kDa}$ ), dysfunction of the endogenous myosin IIB caused similar activity-dependent reduction in synaptic transmission (Fig. $2 A, B, D, E$ ). Phosphorylation by casein kinase II (CKII) or PKC at the C-terminal end of the heavy chain inhibits assembly of IIB in vitro (Murakami et al., 1998). Replacement of serine and threonine at the identified CKII and PKC sites with negatively charged amino acids (CK-D and PK-D) mimics persistent phosphorylation by these kinases and inhibits assembly of IIB into filaments in vitro, while alanine mutations at the CKII sites (CK-A) displayed assembly properties similar to that of IIB-wt (Murakami et al., 2000). Here, injected CK-A mutant into presynaptic SCG neurons produced a slightly greater reduction in the EPSP amplitude, whereas the CK-D and PK-D mutants caused no discernable reduction in synaptic trans- 
A Myosin IIB-wt
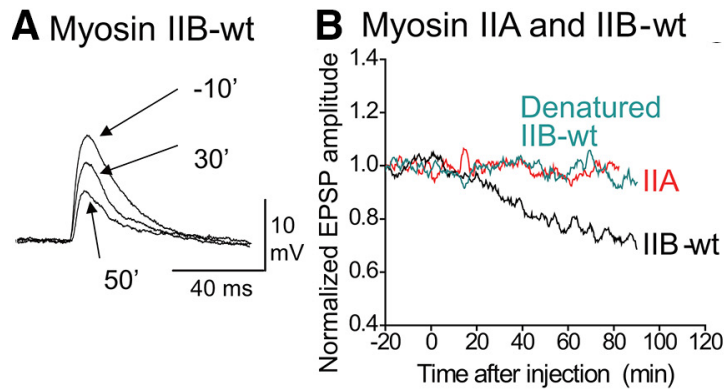

C Myosin IIB-wt and mutants D Myosin IIB-wt

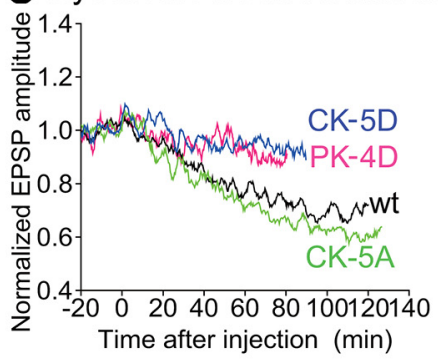

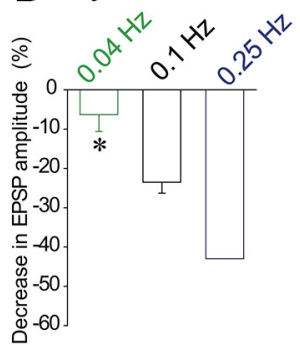

E Myosin IIB-wt

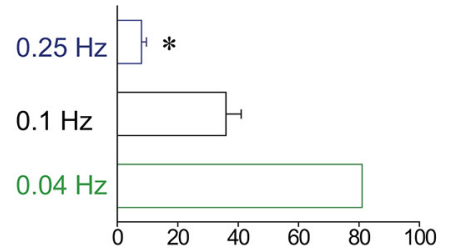

Time requirement for $15 \%$ reduction in EPSP ( $\mathrm{min}$ )

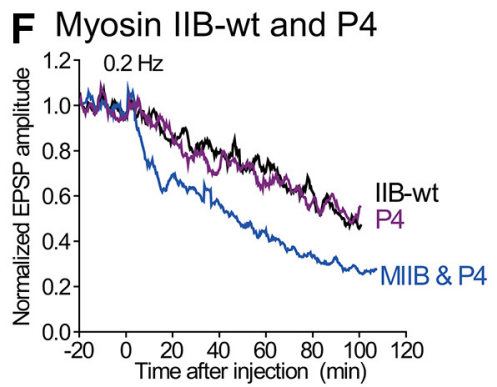

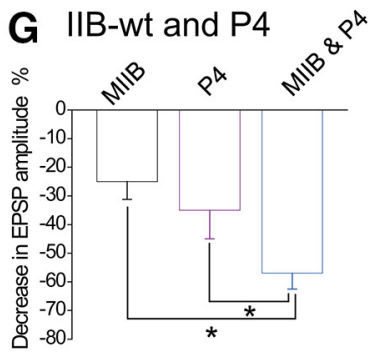

H with $0.1 \mathrm{~Hz}$ stimulation

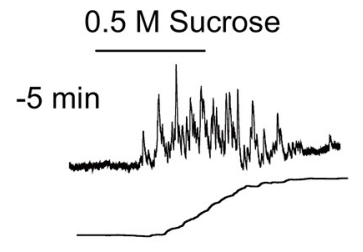

I with $0.1 \mathrm{~Hz}$ stimulation

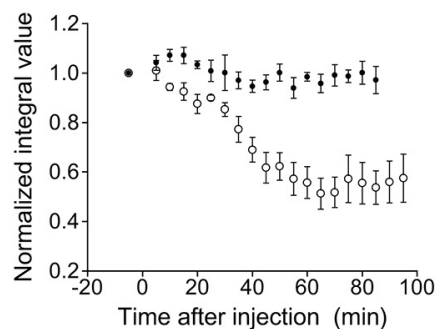

Myosin IIB-wt

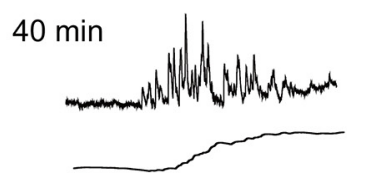

$\mathbf{J}$ without stimulation
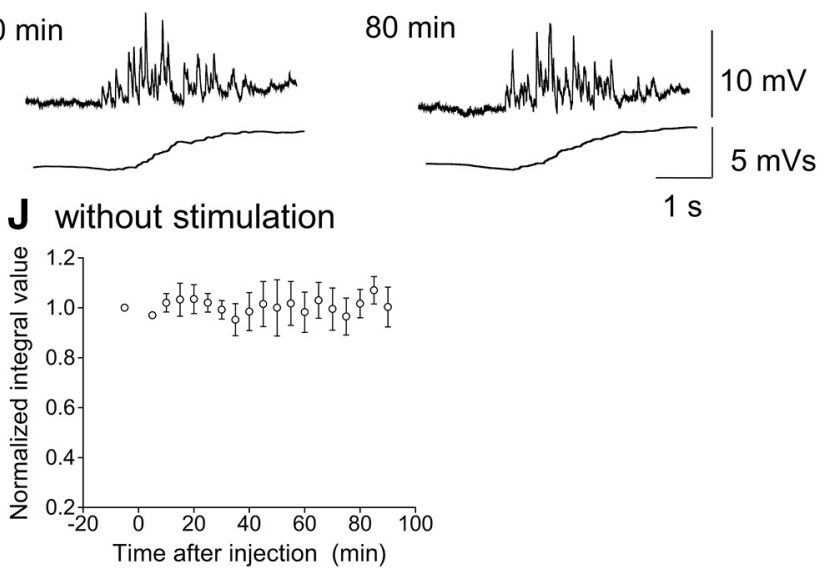

Figure 2. Myosin IIB, but not IIA, fragments inhibit synaptic transmission in a presynaptic activity-dependent manner. Myosin II heavy chain fragments, IIA (46 kDa) or IIB-wt (47 kDa), were injected into presynaptic neurons at time $=0$, at a concentration in the injection pipette of $94 \mu \mathrm{M}$. Presynaptic neurons were stimulated at $0.1 \mathrm{~Hz}$. $\mathbf{A}-\boldsymbol{C}$, Myosin IIB, but not IIA, fragments inhibit synaptic transmission. $\boldsymbol{A}$, EPSPs from one representative experiment. IIB-wt was injected. $\boldsymbol{B}, \boldsymbol{C}$, Normalized EPSP amplitudes are averaged. The resultant values are smoothed with a moving average algorithm and plotted with a line against recording time. $\boldsymbol{B}, \| \mathrm{A}$ (red; $n=5$ ) or IIB-wt (black; $n=6$ ) were injected. As a control, denatured IIB-wt was injected (teal; $n=5$ ). $\boldsymbol{C}$, The IIB fragments mutated at the PKC or CKII sites were injected ( $n=5$ for each experiment). CK-5D (blue) or CK-5A (green): five CKII phosphorylation sites were mutated to Asp or Ala. PK-4D (pink): four PKC phosphorylation sites were mutated to Asp. For comparison, EPSP values with IIB-wt shown in $\boldsymbol{B}$ are also plotted (black). D, $\boldsymbol{E}$, Myosin IIB fragments inhibit synaptic transmission in a presynaptic activity-dependent fashion. D, Bar graph illustrating the decrease in EPSP amplitude at 60 min after IIB-wt injection. At $0.25 \mathrm{~Hz}$ stimulation, carrier solution alone caused a gradual decrease in EPSP amplitude. Therefore, the reduction in EPSP amplitudes with carrier solution alone was subtracted from that with IIB-wt. The net reduction in EPSP amplitude for IIB-wt is illustrated for $0.25 \mathrm{~Hz}$ stimulation. $\boldsymbol{E}$, Bar graph illustrating the mean time \pm SEM for producing a $15 \%$ reduction in EPSP amplitudes. For the $0.04 \mathrm{~Hz}$ stimulation, the mean time producing a $15 \%$ reduction in averaged EPSP amplitudes is illustrated because a $<15 \%$ reduction during $2 \mathrm{~h}$ of recording was observed in 3 of 5 synapses. Asterisk, statistically significant from $0.1 \mathrm{~Hz}$ stimulation at $p<0.05$, with unpaired $t$ test. $\boldsymbol{F}, \mathbf{G}$, Dynamin peptide P4 (1 mm in the injection pipette), which disrupts its interaction with amphiphysin, inhibits synaptic transmission similarly to the myosin IIB fragments but additively inhibits along with IIB-wt, suggesting that myosin IIB acts in the amphiphysin-dependent endocytic pathway but at a different site. $\boldsymbol{F}$, Normalized EPSP amplitudes are averaged. The resultant values are smoothed with a moving average algorithm and plotted with a line against recording time. IIB-wt (black; $n=7$ ), P4 (purple; $n=6$ ), or IIB-wt plus P4 (blue; $n=7$ ) were injected. G, Bar graph illustrating decrease in EPSP amplitude at $40 \mathrm{~min}$ after the injection. Mean \pm SEM. Asterisk, statistically significant with unpaired $t$ test. $\boldsymbol{H}-\boldsymbol{J}$, RRP size is reduced by repetitive stimulation in the presence of myosin IIB fragments. The RRP size was measured by puff application of $0.5 \mathrm{~m}$ sucrose for 2 s every 5 min. $\boldsymbol{H}$, The responses to sucrose applications from one representative experiment recorded 5 min before, 40 min after, and 80 min after IIB-wt injection are illustrated (upper traces) and integrated traces are shown beneath the sucrose response traces. $I, J$, Normalized and averaged integral values for the sucrose responses are plotted $(n=4)$. Presynaptic neurons were stimulated at $0.1 \mathrm{~Hz}$ in $I$, but not in $J$. As a control, integral values for sucrose responses recorded from noninjected synapses are plotted in $I(\bullet, n=5)$.

mission (Fig. 2C). Importantly, synaptic transmission was reduced only by unphosphorylated forms of IIB fragments, and not by IIA fragments (Fig. 2B) or phosphorylated forms of IIB fragments, which are defective for filament assembly. These results suggest that the observed reduction in EPSP amplitude by IIB fragments was due to dominant-negative suppression of endogenous myosin IIB function via the formation of cofilaments with the exogenous recombinant proteins.

Furthermore, the time course and the degree of EPSP reduction by IIB fragments was similar to that of dynamin dysfunction 

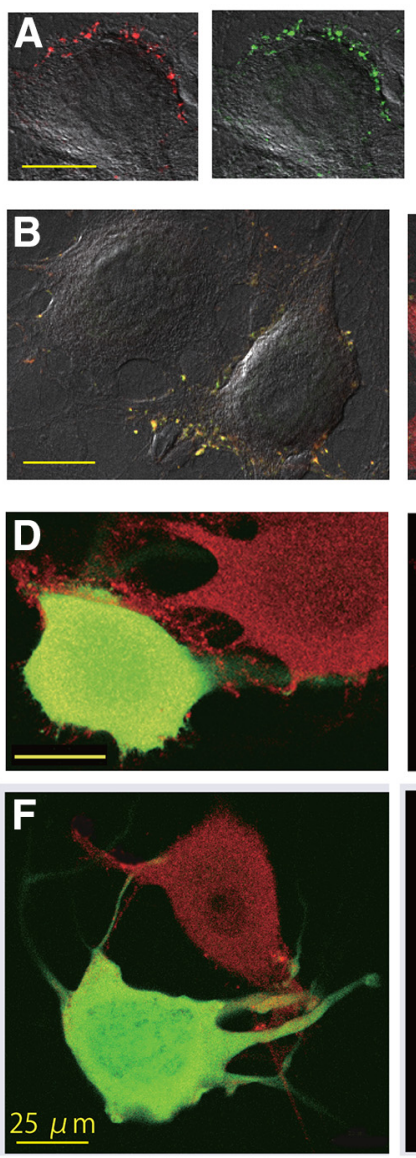
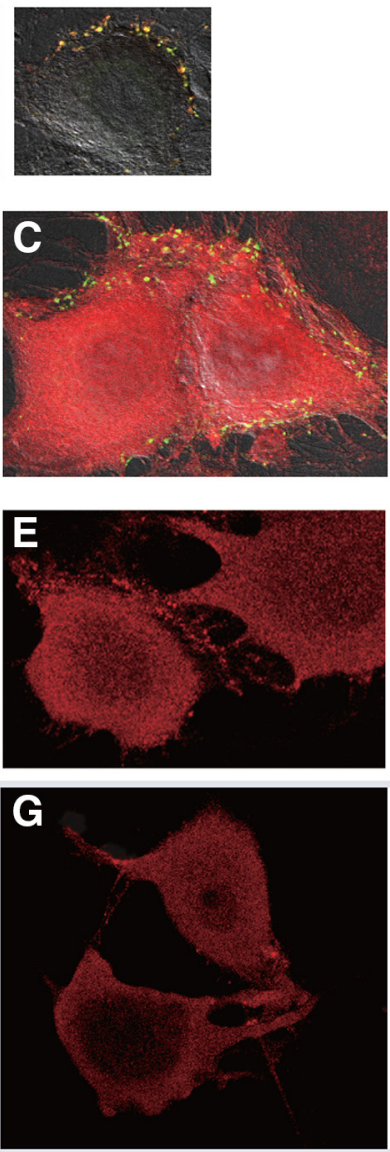

H

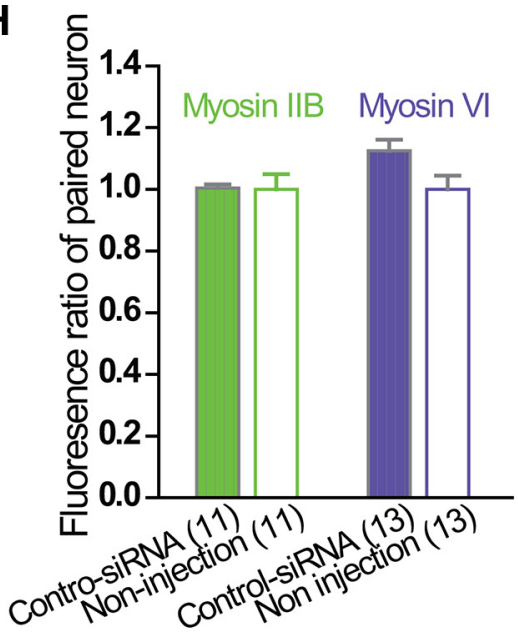

I

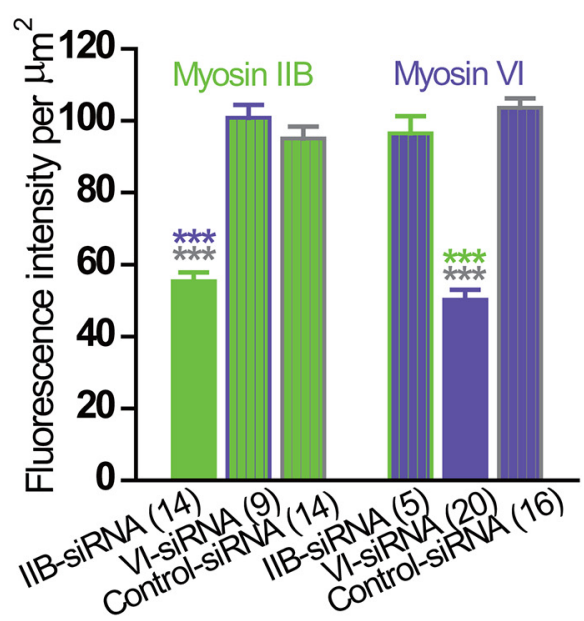

Figure 3. Specificity of myosin siRNAs and antibodies and knockdown of myosin IIB or VI in SCG neurons. A-C, DIC images of 5 weeks' cultured SCG neurons double labeled with anti-myosin IIB (red) and anti-synaptotagmin-1 (green) antibodies ( $\boldsymbol{A}, \boldsymbol{B})$ or with anti-myosin VI (red) and anti-synaptotagmin-1 (green) antibodies (C). D-I, SCG neurons were injected with control-siRNA, myosin IIB-siRNA, or VI-siRNA along with EGFP-DNA, a marker for siRNA injection. $\boldsymbol{D}, \boldsymbol{F}$, Immunofluorescence image of myosin VI (red) and EGFP image (green) of control-siRNA (D) or VI-siRNA injection ( $\boldsymbol{F}$ ). $\boldsymbol{E}, \boldsymbol{G}$, Immunofluorescence image of myosin $\mathrm{VI}(\mathrm{red})$ shown in $\boldsymbol{D}$ and $\boldsymbol{F}$. $\boldsymbol{H}$, Fluorescence intensity of myosin IIB or VI in the processes injected with the control-siRNA was normalized to an adjacent, paired noninjected neuron (mean with SEM, $n=11$ or 13 pairs). I, Fluorescence intensity of myosin IIB or VI in the processes of IIB-siRNA-injected, VI-siRNA-injected, or control-siRNA-injected neurons (mean with SEM, $n=5-20$ ). ${ }^{* * *} p<0.001$; unpaired Student's $t$ test, IIB-siRNA (green) vs VI-siRNA (stripe violet) or control-siRNA-injected neurons (stripe gray), VI-siRNA (violet) vs IIB-siRNA (stripe green), or control-siRNA-injected neurons (stripe gray).

with P4 (QVPSRPNRAP), a dynamin peptide, but was enhanced with coinjection of P4 (Fig. $2 F, G$ ), suggesting that myosin IIB aids SV resupply into the RRP at a site in a dynaminamphiphysin-mediated recycling pathway.

We further examined the myosin VI participation in RRSV resupply into the RRP. Anti-VI antibody also gradually decreased the EPSP over the 50 min recording (Fig. 1C,F). In contrast to IIB dysfunction, the decrease in EPSP amplitude at 40 min after the VI antibody injection (Fig. $1 G$ ) and the time course of the decay were similar between both firing rates (Fig. $1 \mathrm{H}, \mathrm{I}$ ), suggesting an activity-independent action for myosin VI. These results suggest that myosin IIB and VI individually participate in the RRP resupply.

\section{Expression and knockdown of IIB and VI in SCG neurons}

We examined the quantitative protein distribution of myosin IIB and VI in SCG neurons and assessed their equivalent genetic knockdown by siRNA. We have shown that myosin IIB is expressed in the presynaptic terminals and colocalizes with an SV protein synaptophysin (Wiedenmann and Franke, 1985) or an active zone protein bassoon (tom Dieck et al., 1998; Takagishi et al.,
2005). Here, we show that IIB colocalizes with synaptotagmin-1 (Fig. $3 A, B$ ) and distributes similarly to VI-expressed surrounding cell bodies (Fig. $3 C, D$ ), confirming that $\mathrm{VI}$ is also expressed in the presynaptic terminals. To quantitatively assess the reduction of myosins IIB and VI by each siRNA, the averaged fluorescence intensity of IIB and VI in five randomly chosen regions of 10 $\mu \mathrm{m}^{2}$, each containing two to three dots on the EGFP coexpressed processes, was compared with the averaged value of regions from control siRNA-injected neurons (Fig. 3D). The ratio of fluorescence intensity of IIB and VI in control siRNA-injected neurons to adjacent noninjected neurons is $\sim 1$ (Fig. $3 E, H$ ), suggesting that myosin IIB and VI expression levels were not different between noninjected neurons and control siRNA-injected neurons and that dialysis of negative siRNA has no effect on myosin expression level. Indeed, VI and IIB siRNA did not reduce fluorescence intensity of IIB and VI, respectively (Fig. 3I), while each siRNA significantly reduced the corresponding myosins (Fig. $3 G, I$ ) within the standard range for reduction of protein level with siRNA knockdown (Su et al., 2004; Tanifuji et al., 2013; Vogl et al., 2015). Importantly, each isoform-specific siRNA did not reduce the expression levels of other myosin (Fig. 3I), suggesting 

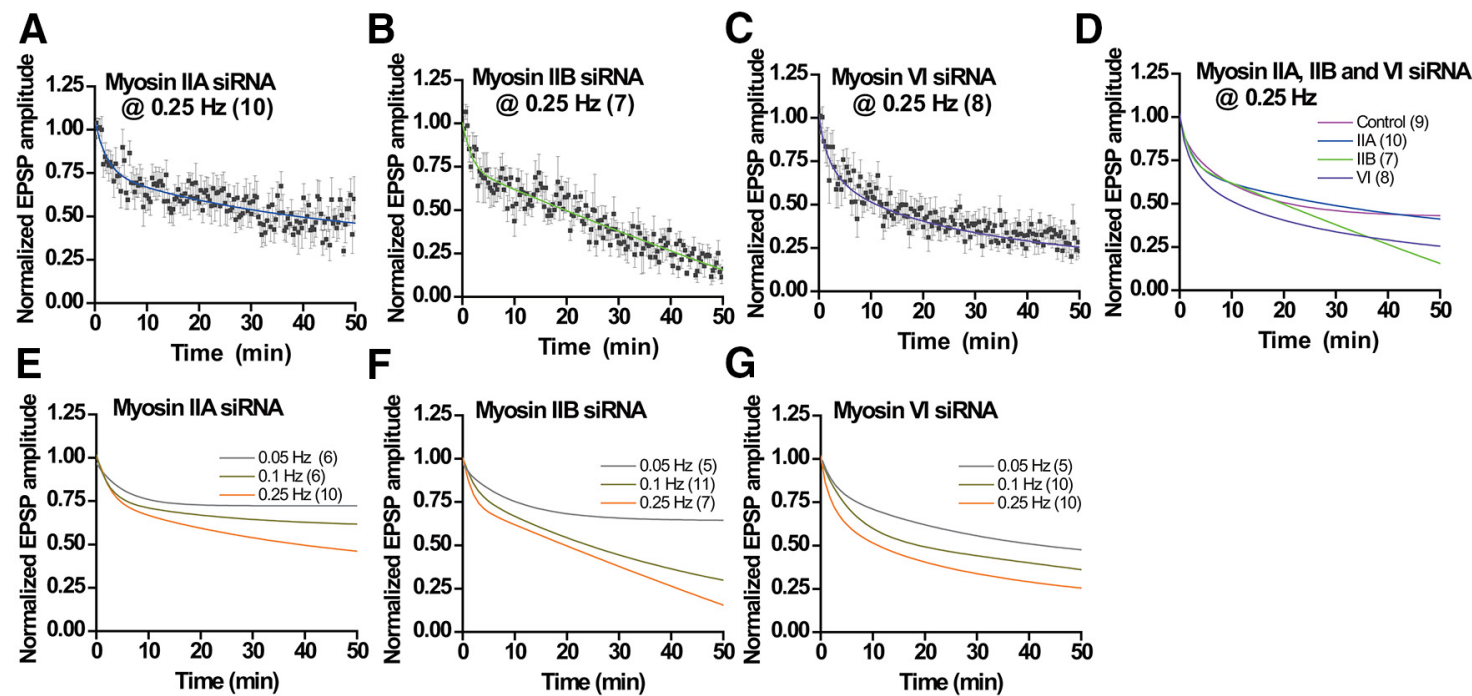

Figure 4. Myosin IIB or VI loss of function impairs RRSVs during low-frequency transmitter release. Presynaptic neurons were injected with control, myosin IIA-siRNA, IIB-siRNA, or VI-siRNA 2-3 $\mathrm{d}$ before the EPSP recordings. $\boldsymbol{A}-\boldsymbol{C}$, Normalized and averaged EPSP amplitudes with SEM recorded at $0.25 \mathrm{~Hz}(n=7-9)$ and the double exponential curves. EPSP amplitudes were normalized to the mean of the first three EPSP amplitudes $(n=7-10)$. D, The exponential curves shown in $\boldsymbol{A}-\boldsymbol{C}$ and that for EPSP amplitudes recorded in the control-siRNA-injected neurons. $\boldsymbol{E}$ - $\mathbf{G}$, The exponential curves for EPSP amplitude reduction with myosin IIA-siRNA $(\boldsymbol{E})$, IIB-KD $(\boldsymbol{F})$, and VI-KD $(\boldsymbol{G})$ recorded at $0.05,0.1$, and $0.25 \mathrm{~Hz}$.

that knockdown of IIB and VI by each siRNA is selective and that individual myosin siRNA does not produce off-target effects.

\section{Myosin IIB and VI loss of function under low-frequency AP firing}

Using a genetic approach with their specific siRNA transfection, we further confirmed individual participation of myosin II and VI in the RRP resupply (Fig. 4). Control-siRNA or IIA-siRNA dialysis showed gradual decrease in RRSVs over the 50 min recording in response to consecutive AP firing at $0.25 \mathrm{~Hz}$ (Fig. $4 A, D)$. Knockdown (KD) of IIB or VI showed more rapid decrease in RRSVs (Fig. $4 B, C$ ). The results of myosin II-KD, induced by specific siRNA (Fig. 4), were consistent with those of their dysfunction induced by a specific antibody (Fig. 1) or the recombinant heavy chain fragments (Fig. 2A-G). Myosin VI-KD rapidly decreased the EPSP amplitude with presynaptic AP firing at a low frequency of $0.25 \mathrm{~Hz}$ (Fig. 4C), while IIB-KD caused additive decrease in EPSP amplitude following consecutive presynaptic APs (Fig. 4B). The time course of RRSV reduction with IIA-siRNA was similar to that with control-siRNA, while the reduction time course with IIB-KD or VI-KD was significantly different from each other, and control-siRNA or IIA-siRNA dialyzed presynaptic neurons (Fig. $4 D$; one-way ANOVA, $p<$ 0.0001 ). In addition, the reduction time courses for $0.05,0.1$, and $0.25 \mathrm{~Hz}$ were significantly different in control-siRNA (data not shown; Tanifuji et al., 2013) or IIA-siRNA dialysis (Fig. $4 E ; p<$ 0.001 , Bonferroni post hoc test after one-way ANOVA), suggesting that the general reduction rate of RRSVs is AP firingfrequency dependent (Mochida et al., 1994a). At each AP firing frequency, reduction time courses for IIB-KD and VI-KD are significantly faster than that for IIA-siRNA dialysis (Fig. $4 E-G$; $p<0.05$, Bonferroni post hoc test after one-way ANOVA). Furthermore, at a lower frequency of $0.05 \mathrm{~Hz}$, the reduction was greater with VI-KD than IIB-KD, while it was more rapid with IIB-KD than VI-KD at 0.1 and $0.25 \mathrm{~Hz}$. Together with the results of antibody-induced or the recombinant $\mathrm{HC}$-induced myosin dysfunction, our siRNA data demonstrate that myosin IIB is required for the RRP resupply related to consecutive AP firings and the rate. In addition to myosin IIB, at presynaptic terminals of sympathetic neurons, myosin VI also supports the RRP resupply in a firing rate-independent manner.

\section{The RRP resupply mediated by myosin IIB and VI under high-frequency AP firing}

To further examine the relationship of myosin IIB and VI to AP firing activity, their effect on the RRP resupply during and after a high rate of repetitive transmitter release was examined. For this purpose, $2 \mathrm{~s} \mathrm{AP}$ trains at 5, 10, and $20 \mathrm{~Hz}$ were applied every $2 \mathrm{~min}$ to the presynaptic neurons having knocked down myosin IIB or VI. Similar to nontransfected neurons (Lu et al., 2009), AP trains with a high frequency of $20 \mathrm{~Hz}$ induced synaptic depression with control-siRNA or IIA-siRNA dialysis (Fig. 5A; averaged EPSP traces of control and IIA). In contrast, with IIB-KD or VI-KD, severe synaptic depression was extended to lower frequencies of 5 $\mathrm{Hz}$ or $10 \mathrm{~Hz}$, in addition to $20 \mathrm{~Hz}$ (Fig. $5 \mathrm{~A}$, IIB or VI). Two minutes after a $5 \mathrm{~Hz}$ or $10 \mathrm{~Hz}$ AP train, RRSV was recovered from depression, suggesting that the RRP resupply completes within 2 min with or without IIB and VI participation. It is unlikely that the RRP size was affected by IIB or VI-KD under $\leq 10 \mathrm{~Hz}$ short ( 2 s) AP firing, because the number of SVs in the RRP [see Materials and Methods; estimated from the back-extrapolation (to time $=$ 0 ) of cumulative EPSP amplitudes recorded at $10 \mathrm{~Hz}$; Schneggenburger et al., 1999; Inchauspe et al., 2007] was unchanged with IIA-siRNA, IIB-siRNA, and VI-siRNAs (100, 96, and 103 SVs, respectively), or control-siRNA (105 SVs) (Fig. 5C, $10 \mathrm{~Hz}$ ). However, with an AP train at $20 \mathrm{~Hz}$, the RRP size of IIB-KD or VI-KD (IIB, 153 SVs; VI, 143 SVs) was smaller than those of controls (control, 165 SVs; IIA-KD, 168 SVs) (Fig. 5C, 20 Hz). Myosin IIB dysfunction did not affect the RRP size measured by $0.5 \mathrm{M}$ sucrose application, but reduced it with repetitive AP firing (Fig. $2 \mathrm{H}-J$ ). These results suggest that both IIB and VI support the RRP resupply during higher levels of synaptic activity. Interestingly, although the time course of the peak amplitude reduction after the third EPSP in a train of $5 \mathrm{~Hz}$ or $10 \mathrm{~Hz}$ was comparable between IIB and VI, the second EPSP amplitude was more sensitive to VI-KD (Fig. 5B), suggesting that VI acts fast with an AP generation. A higher frequency such as $20 \mathrm{~Hz}$, which induces $\mathrm{Ca}^{2+}$ channel-mediated synaptic depression (Mochida et al., 2008), 
A

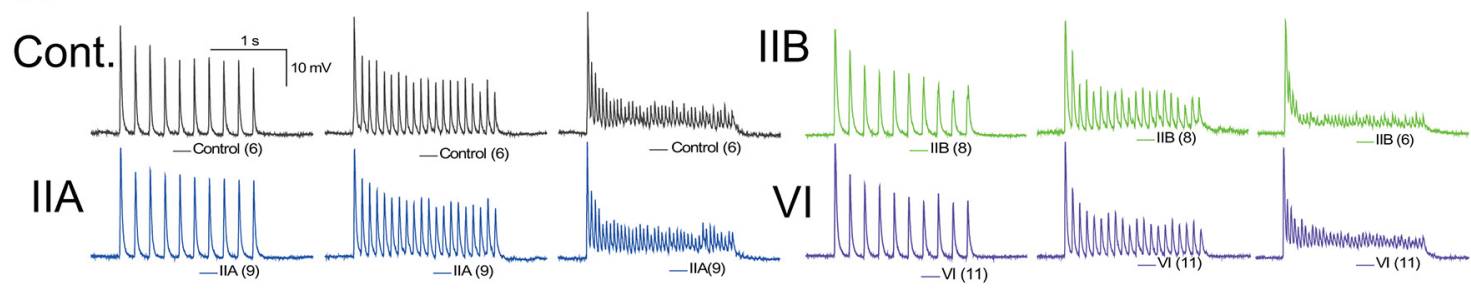

B
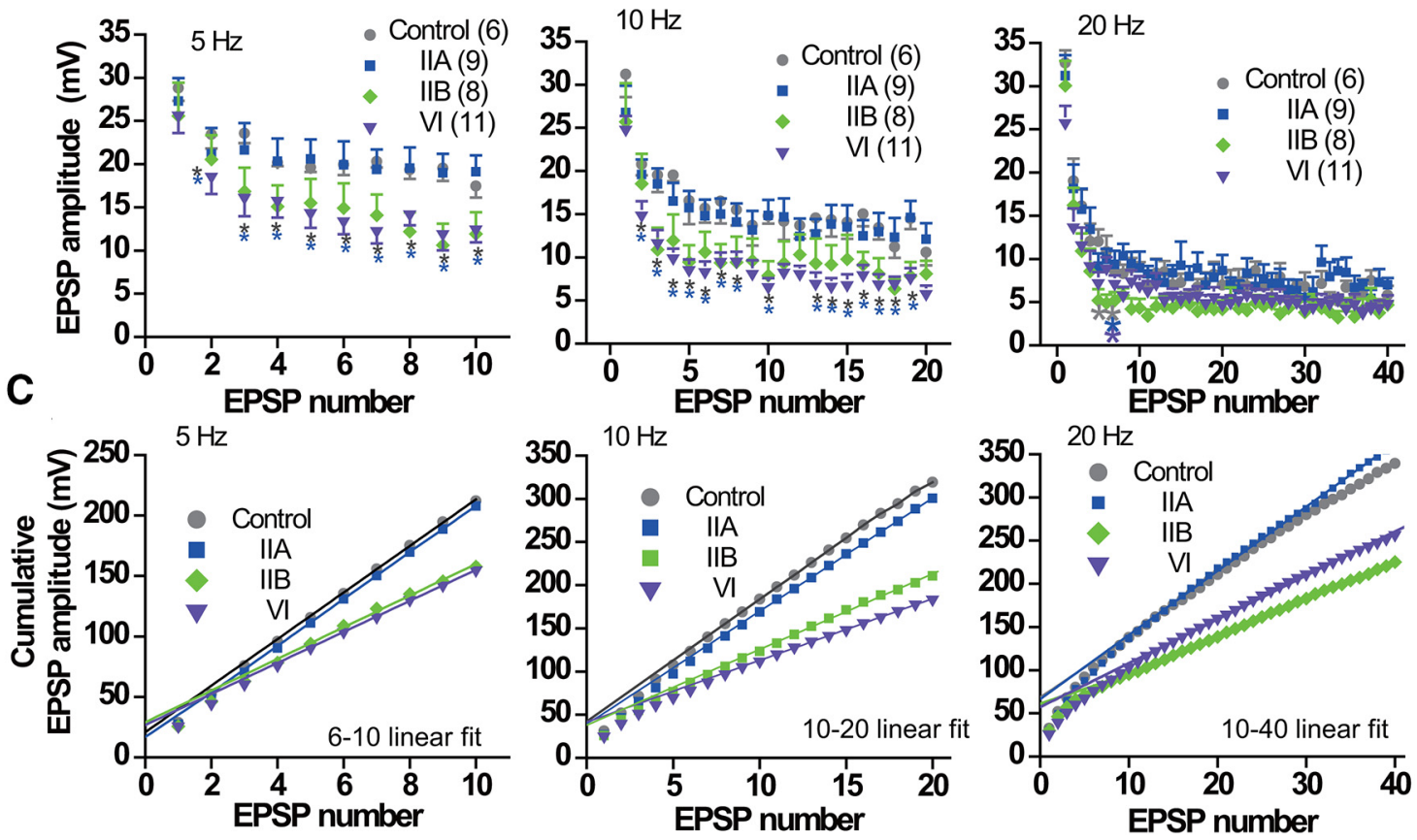

Figure 5. Myosin IIB or VI loss of function reduces RRSVs during and after high-frequency transmitter release. Presynaptic neurons were injected with control-siRNAs or myosin IIA-siRNAs, IIB-siRNAs, or VI-siRNAs (Cont., IIA, IIB, or VI) as in Figure 4. The 2 s AP trains at 5, 10, and $20 \mathrm{~Hz}$ were used to elicit EPSPs; three recordings every 2 min were performed for each frequency ( $n=6-11$ ). $A$, Averaged EPSP traces from consecutive recordings of $6-11$ neuron pairs. $B$, Averaged EPSP amplitudes with SEM plotted against the EPSP number. ${ }^{*} p<0.05$; unpaired $t$ test, vs control (gray), vs IIA (blue), or vs VI (violet). C, Cumulative EPSP amplitudes recorded at 5, 10, and $20 \mathrm{~Hz}$ were plotted and back-extrapolated to time 0 to estimate the RRP size. $p<0.001$, Bonferroni post hoc test after one-way ANOVA, control or IIA vs IIB or VI; $p>0.05$, Bonferroni post hoc test after one-way ANOVA, control vs IIA and IIB vs VI.

showed synaptic depression with control-siRNA or IIA-siRNA and caused stronger depression with IIB-KD (Fig. 5A,B), indicating that IIB can drive the RRP resupply during a higher rate of synaptic activity. These results suggest that myosin IIB and VI differentially contribute to maintain the RRP via replenishment of SVs during high-frequency transmitter release.

\section{Myosin IIB and VI contribution immediately after single AP firing}

We next examined the relationship of myosin IIB and VI to AP properties for the kinetics of vesicle resupply. IIB or VI in presynaptic neurons was knocked down, and the recovery of RRSVs after evoked transmission was assessed with a paired-pulse protocol (Fig. 6). Similar to nontransfected neurons (Lu et al., 2009; Magupalli et al., 2013; Tanifuji et al., 2013), synapses dialyzed with IIA-siRNA showed depression of the second EPSP (pairedpulse depression, PPD) at short ISIs ( $\leq 100 \mathrm{~ms}$ ), while the amplitudes of the second EPSP were similar to that of the first EPSP at longer ISIs ( $\geq 100 \mathrm{~ms}$; Fig. $6 A, B$ ). IIB-KD unchanged the second EPSP amplitude at all ranges of ISIs (20-200 ms), which is consistent with the results of IIB dysfunction induced by heavy chain fragments (Fig. 8A). In contrast, VI-KD increased PPD at the ISI of 50-200 ms (Fig. 6A,B). It is unlikely that basal release proba- bility is affected by VI-KD in nonactive SCG neurons in culture (Mochida et al., 1994b) because mean EPSP amplitudes of the first recording were unchanged with VI-siRNA dialysis (Fig. 6A). Thus, myosin VI contributes to regulation of rapid recovery of RRSVs in the RRP, which occurs within 50 ms after transmitter release is evoked by an AP. However, IIB does not appear to contribute significantly to the rapid regulation of RRSVs after single firing, confirming an asymmetry in the involvement of myosin IIB and VI in the major kinetic modes of the RRP resupply.

\section{Myosin IIB and VI regulation in dynamin-mediated recycling pathways}

We next asked how myosin IIB and VI contribute to SV recycling pathways with distinct kinetics activated by dynamin isoforms (Tanifuji et al., 2013). Previously we showed that after depletion of RRSVs the SV pool recovers at two time constants, fast and slow, through distinct pathways (Lu et al., 2009). To map IIB and VI specificity onto the fast and slow components we applied the same recovery protocol. The recovery of RRSVs following full depletion of the SV pool due to a train of 4 min APs at $5 \mathrm{~Hz}$ was monitored by measuring the EPSP amplitude every $1 \mathrm{~s} \mathrm{(Fig.} \mathrm{7A-}$ $E$ ). To determine IIB and VI effects on fast and slow recovery, we 
measured EPSP amplitudes at $5 \mathrm{~s}$ (during the fast recovery) and 6 min (during the slow recovery) after cessation of the AP train (Fig. $7 F$ ). Each myosin-KD appeared to prevent recovery in both cases; however, reduction in the EPSP amplitude was significant for IIB-KD at $5 \mathrm{~s}$ and $6 \mathrm{~min}$ and for VI-KD at $6 \mathrm{~min}$. These results suggest myosin IIB and VI specificity on the fast and slow components. Therefore, we further analyzed the kinetics associated with IIB and VI in the regulation of the fast and slow SV recycling pathways. We compared recovery time constants with exponential growth curves fitted to the increase in the mean EPSP amplitude (Fig. 7M). Control-siRNA and IIA-siRNA showed a time constant for fast $(\tau=7.5 \pm 1.8$ and $8.4 \pm 1.9 \mathrm{~s}$, respectively, $n=8,5)$ and slow recovery $(\tau=$ $3.6 \pm 0.7$ and $5.5 \pm 1.3 \mathrm{~min}$, respectively, $n=8,5$, recovery time constant by $8 \mathrm{~min}$ after depletion). The curves for VI-KD showed a similar time course of the fast recovery to control-siRNA and IIA-siRNA, while that of IIB-KD showed moderate recovery (Fig. 7M). To obtain a clearer comparison of the fast recovery rate, exponential curves by $15 \mathrm{~s}$ are plotted (Fig. $7 N$ ). This indicated significant delays of fast recovery (13.6 $\pm 3.4 \mathrm{~s}, n=13)$ with IIB-KD; the degree of slow recovery was clearly much less than that of the control (Fig. 7M), whereas the relative recovery rate of normalized EPSP amplitude to the initial phase of slow recovery (0.51$0.6 \mathrm{~min}$ ) was similar to those of control-siRNAs and IIA-siRNAs (Fig. 7O). In the presence of the IIB fragments, the fast recovery phase after the RRP deletion by either an AP train or $0.5 \mathrm{M}$ sucrose application was delayed (Fig. $8 B-E$ ). In contrast, with VI-KD the fast recovery phase was faster $(\tau=2.1 \pm 0.7 \mathrm{~s}, n=8)$ and reached a plateau by $6.5 \mathrm{~s}$ (Fig. $7 \mathrm{~N}$ ), and the slow recovery phase was delayed (Fig. $7 \mathrm{M}, \mathrm{O}$ ). Furthermore, double KD of IIB and VI (Fig. $7 G$ ) indicated significant delays of fast and slow recovery phases (Fig. 7M). Thus, a kinetic analysis suggests myosin IIB and myosin VI specificity for fast and slow recycling pathways.

Previously we showed that the fast and slow recovery phases of RRSVs are mediated through distinct pathways involving endocytosis activated by dynamin-1 and dynamin-3, respectively (Tanifuji et al., 2013). Thus, we subsequently analyzed the participation of myosin IIB and myosin VI in the two distinct SV recycling pathways. The contribution of individual isoforms to the fast and slow components could be confirmed in a double KD of dynamin- 1 or dynamin-3 and myosin IIB or myosin VI, which resulted in additive effects or similar effects on single KD. Each dynamin siRNA reduced individual dynamins within the similar range with myosin siRNA knockdown (Tanifuji et al., 2013). Interestingly, double KD severely delayed RRSV recovery (Fig. 7H$J$ ), except for dynamin-3 and myosin VI double KD (Fig. $7 \mathrm{~K}$ ), suggesting that dynamin-3 and VI, but not dynamin-1 and IIB, dynamin-1 and VI, or dynamin-3 and IIB, act at a similar site in $\mathrm{SV}$ recycling pathways. Although the degree of RRSV recovery is smaller (Fig. 7L), dynamin-1 and IIB double KD did not affect the fast recovery rate with IIB-KD, and dynamin- 1 and VI double $\mathrm{KD}$ delayed both fast and slow recovery (Fig. $7 M, N$ ). In addition, dynamin-3 and IIB double KD delayed both fast and slow recovery (Fig. $7 M, N)$. In contrast, dynamin-3 and VI double KD did not affect both recovery rates with VI-KD (Fig. $7 M, N)$. These

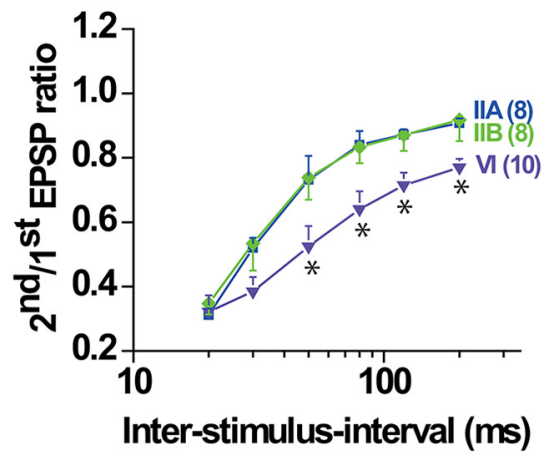

B

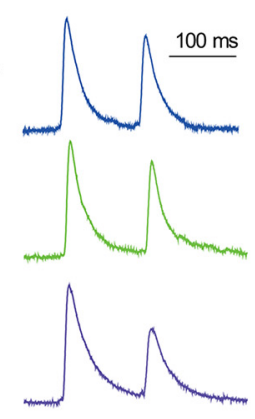

Figure 6. Myosin VI loss of function reduces RRSVs after AP-evoked transmitter release. Presynaptic neurons were injected with myosin IIA-siRNAs, IIB-siRNAs, or VI-siRNAs (IIA, IIB, or VI) as in Figure 4. Changes in EPSP amplitude after an AP-evoked transmitter was monitored by eliciting two consecutive APs at various ISIs, every $1 \mathrm{~min}$, in an siRNA-injected neuron. Three recording veraged paired-pulse ratio with SEM is plotted against the ISI. $p<0.05$; Bonferroni post hoc test after one-way ANOVA. VI-KD vS

results suggest that myosin IIB and VI have a distinct relationship to fast and slow SV recycling pathways mediated by dynamin-1 and dynamin-3 activations to replenish a common SV pool.

\section{Discussion}

\section{AP firing pattern selects myosin IIB and VI in distinct vesicle} resupply mode

Myosin II and VI are actin-based cytoskeletal motors that drive actin dynamics and membrane transport in the brain (Kneussel and Wagner, 2013). So far, no evidence for their regulation of ion channels or transporters (which control SV pools), SV release probability, and quantal size has been provided. Thus, we can exclude confounding factors that influence EPSP size and quantify SV resupply to the RRP and recovery of the RRSVs after the consumption with AP firing by measuring postsynaptic EPSP.

In this study we first demonstrate presynaptic roles of myosin VI, in addition to myosin IIB, as SV recycling motors, and address the fundamental issue of how the SV recycling motors can adapt to wide variations in AP frequency. Our overall findings indicate that myosin IIB and VI together cover the range of AP frequency patterns for the selection of specific vesicle resupply modes that replenish a shared RRP through distinct endocytic pathways mediated by each dynamin isoform. Resupply of the RRP via a myosin IIB-mediated pathway occurred both during and after consecutive AP firing (0.1-20 Hz; Figs. 1, 4, 5). In contrast, myosin VI mediated a separate mode of RRP resupply that was independent of AP frequency (Figs. 1, 4) and activated rapidly ( $\leq 0.05 \mathrm{~s})$ after AP generation (Fig. 6). AP firing pattern-dependent and patternindependent modes of the RRP replenishment are activated through dynamin-1-mediated and dynamin-3-mediated endocytosis, respectively, whereas dynamin-2 contributes to both endocytoses, as we previously showed (Tanifuji et al., 2013). Thus, myosins IIB and VI appear to provide coverage of SV resupply required to respond to the full physiological range of AP firing patterns that are decoded by dynamin isoforms via diverse endocytic pathways with distinct rates and time constants

\section{The temporal window for the RRP replenishment}

This study is the first to assign roles to the myosin IIB and VI in replenishment of a shared SV release pool (the RRP) in response to variations in AP firing. Furthermore, our findings suggest that 


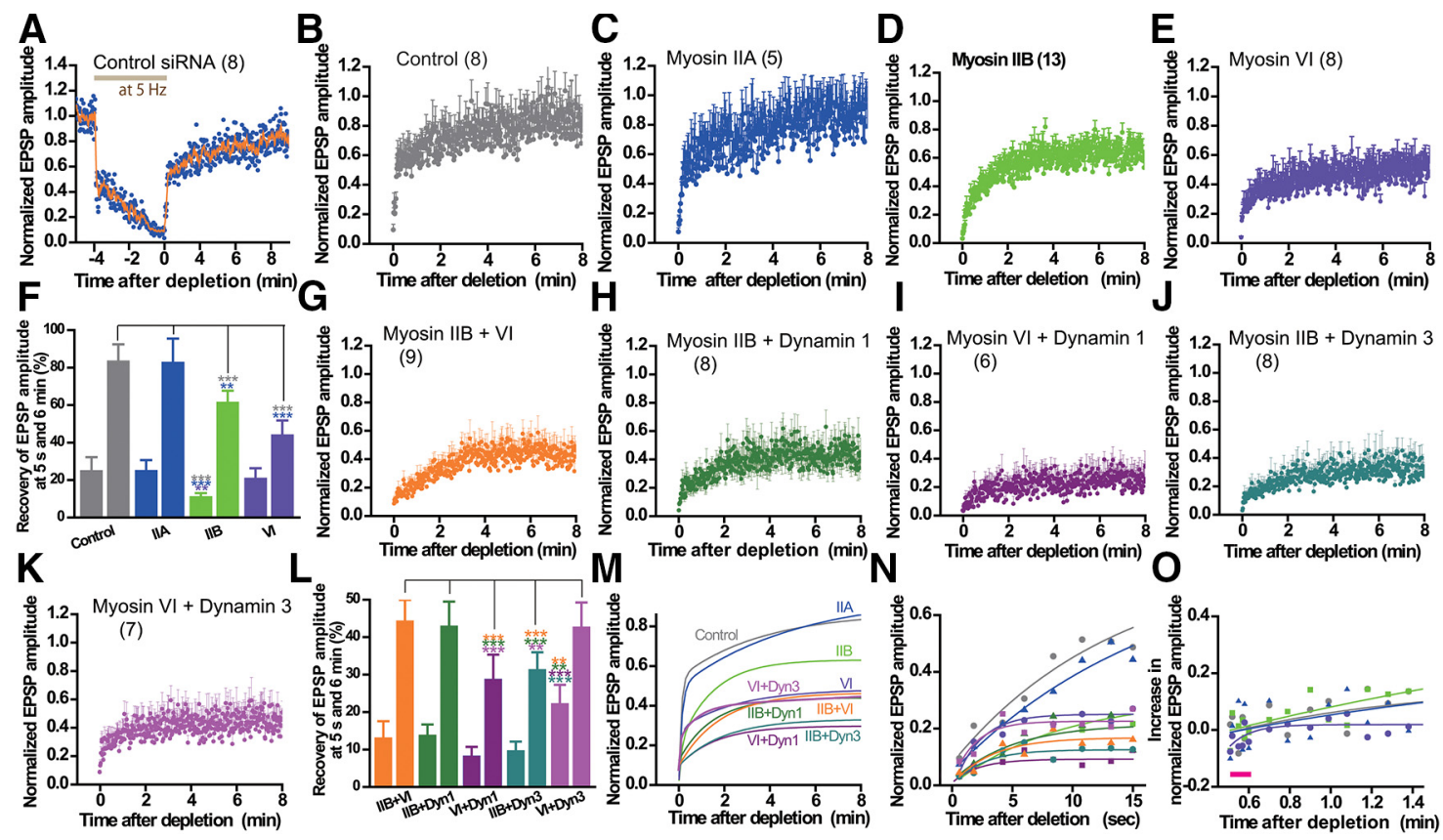

Figure 7. Myosin IIB or VI loss of function delays the recovery of RRSVs. Presynaptic neurons were injected with control-siRNA, myosin-siRNA, or myosin-siRNA plus dynamin-siRNA as in Figure 4. The recovery of RRSVs in a neuron with myosin-KD was monitored by measuring the amplitude of EPSP recorded at $1 \mathrm{~Hz}$. After a $1 \mathrm{~min}$ control recording at $1 \mathrm{~Hz}$, a 4 min $A P$ train at $5 \mathrm{~Hz}$ was applied to deplete SVs. EPSP amplitudes were normalized to the mean EPSP amplitudes before the $4 \mathrm{~min}$ train. $A$, Averaged EPSP amplitudes plotted with the smoothed lines using a moving average algorithm $(n=8) \cdot \boldsymbol{B}-\boldsymbol{E}$, Recovery of EPSP amplitudes after the RRP depletion by the AP train $(n=5-13)$. $\boldsymbol{F}$, Recovery of EPSP amplitudes at 5 s (left bar) and $6 \mathrm{~min}$ (right bar) shown in $\boldsymbol{B}-\boldsymbol{E}$. ${ }^{* * *} p<$ $0.001,{ }^{* *} p<0.01$; Bonferroni post hoc test after one-way ANOVA, vs control, IIA-KD, IIB-KD, and VI-KD. G-K, Recovery of EPSP amplitudes after the RRP depletion within double KD of IIB and VI (G), IIB and dynamin-1 $(\boldsymbol{H})$, VI and dynamin- $1(\boldsymbol{I})$, IIB and dynamin-3 $(\boldsymbol{J})$, and VI and dynamin-3 $(\boldsymbol{K} ; \boldsymbol{n}=6-9)$. $\boldsymbol{L}$, Recovery of EPSP amplitudes at 5 s (left bar) and 6 min (right bar) shown in $\mathbf{G}-\boldsymbol{K}$. ${ }^{* * *} p<$ $0.001,{ }^{* *} p<0.01$; Bonferroni posthoc test after one-way ANOVA, vs double KD of IIB and VI, IIB and dynamin-1, VI and dynamin-1, IIB and dynamin-3, and VI and dynamin-3.M, Double exponential growth curves for control, myosin IIA-KD, IIB-KD, VI-KD, and double-KD calculated from $\boldsymbol{B}-\boldsymbol{E}$ and $\mathbf{G}-\boldsymbol{K}$. The noise level value of EPSP recording at time $=0$ shown in $\boldsymbol{A}-\boldsymbol{E} / \mathbf{G}-\boldsymbol{K}$ is subtracted. Bonferroni post hoc test after one-way ANOVA, $p>0.05$, Control vs IIA; $p<0.001$, Control vs the others; $p>0.05$, IIA vs Control; $p<0.001, \|$ vs the others; $p<0.001$, IIB vs VI, IIB and VI, IIB and dymanin-1, IIB and dymanin-3, VI and dymanin-1, VI and dymanin-3; $p<0.001$, VI vs IIB and VI; $p>0.05$, VI vs IIB and dynamin-1; $p<0.001$, VI vs IIB and dynamin-3, VI and dymanin-1; $p>$ 0.05 , VI vs VI and dymanin-3; $p<0.001$, IIB and VI vs IIB and dymanin-1; $p>0.05$, IIB and VI vs IIB and dymanin-3, VI and dynamin-1; $p<0.001$, IIB and VI vs VI and dynamin-3; $p<0.001$, IIB and dynamin-1 vs IIB and dymanin-3, VI and dynamin-1; $p>0.05$, IIB and dynamin-1 vs VI and dynamin-3, IIB and dynamin-3 vs VI and dynamin-1; $p<0.001$, IIB and dynamin-3 vs VI and dynamin-3, VI and dynamin-1 vs VI and dynamin-3. N, The single exponential curves fitted with normalized EPSP amplitudes by $15 \mathrm{~s}$. The averaged noise level value of EPSP recording at time $=0$ is subtracted. Bonferroni post hoc test after one-way ANOVA, $p<0.01$, Control vs VI; $p<0.001$, Control vs IIB, IIB and dynamin-1, IIB and dynamin-3, VI and dynamin-1; $p<0.01, \| \mathrm{A}$ vs IIB, IIB and dynamin-1, IIB and dynamin-3, VI and dynamin-1. 0, The EPSP amplitudes shown in $\boldsymbol{B}-\boldsymbol{E}$ were normalized to averaged values of six points recorded every $1 \mathrm{~s}$ from 0.51 to 0.60 min after the AP train (pink bar) to show the start of the slow recovery phase and relative value of the EPSP amplitude increase. The single exponential curves fitted with normalized EPSP amplitudes. Bonferroni post hoc test after one-way ANOVA, $p<0.01$, Control (gray) or IIA (blue) vs VI (violet); $p<0.001$, IIB (green) vs VI. Error bars indicate SEM.

the cells have intrinsic mechanisms to control the temporal window for vesicle pool replenishment (Fig. 6). In the case of sympathetic neurons, single AP activates RRP replenishment via dynamin-1 in a slow window of $0.05 \mathrm{~s}$ and lasting $5 \mathrm{~s}$, while dynamin-2 and dynamin-3 activation occurs within a faster $0.02 \mathrm{~s}$ window lasting $5 \mathrm{~s}$ and 20-120 s, respectively (Tanifuji et al., 2013). We monitored replenishment rate of the same RRP with myosin IIB-KD and VI-KD. The time window for myosin VI $(0.05 \mathrm{~s}$; Fig. $6 B)$ is slower than that of dynamin-3 (0.02 s; Tanifuji et al., 2013), but both are in a similar timescale range, suggesting that myosin VI may contribute vesicle mobilization following the dynamin-2-mediated and dynamin-3-mediated endocytoses. In contrast, the time window for myosin IIB $(0.2 \mathrm{~s}$; Fig. $5 A, B)$ is much slower than that of dynamin-1 (0.05 s; Tanifuji et al., 2013), and myosin IIB activation requires repetitive AP firing (Figs. 1, 4, 5). In addition, dynamin P4 peptide accelerated the EPSP reduction with IIB dysfunction (Fig. 2). These results suggest that myosin IIB is not required for the dynamin-1-mediated endocytosis by itself in sympathetic neurons. In contrast, myosin II seems to be a critical regulator of the clathrin-mediated endocytosis at central synapses (Chandrasekar et al., 2014); there, myosin II is reported to act on cortical actin scaffolds primarily in the plane of the plasma membrane to generate changes critical for enhancing clathrin-mediated endocytosis progression.

\section{Myosin VI acts as quickly as dynamin}

The speed of endocytosis, ranging from hundreds of milliseconds to hundreds of seconds, has been estimated by capacity measurements and synaptopHluorin imaging ( $\mathrm{Wu}, 2004)$. In contrast to fast endocytosis, a more rapid time window of tens of milliseconds suggests a role for dynamin in the clearance of endocytic vesicle cargo from active zones. A requirement $(<20 \mathrm{~ms}$ after an AP) for rapid dynamin action in SV membrane trafficking and active zone clearance has been proposed for the D. shibire mutant (Kawasaki et al., 2000) and at the calyx of Held after dynamin dysfunction (Hosoi et al., 2009), respectively. In addition, ultrafast endocytosis occurs within 50-100 ms without forming clathrin coats at the site flanking the active zone in mouse hippocampal synapses, which is mediated by actin polymerization and dynamin to restore the surface area of the membrane rapidly (Watanabe et al., 2013, 2014). Our present studies add myosin VI in the dynamin-mediated but amphiphysin-independent rapid vesicle recycling pathway triggered by each AP firing (Lu et al., 2009; Tanifuji et al., 2013). The time window for myosin VI (50 
A
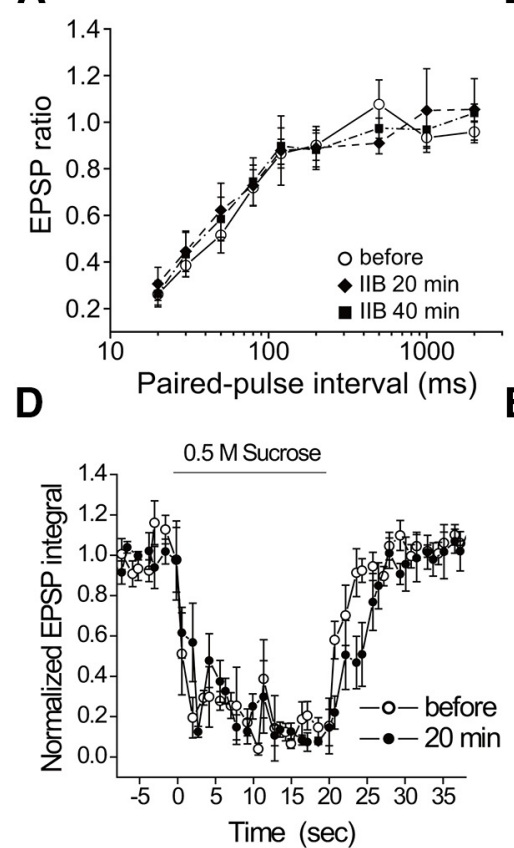

B
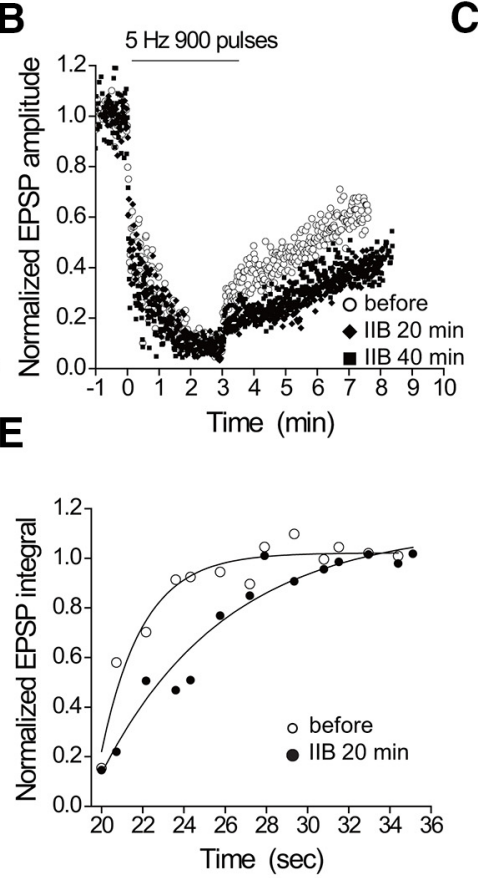

C

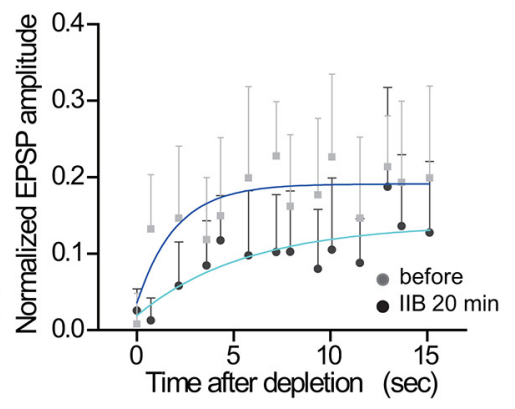

Figure 8. Myosin IIB fragments delayed fast phase of synaptic vesicle replenishment into the RRP. Myosin IIB heavy chain fragments (IIB) were introduced into presynaptic neurons as in Figure 2. EPSPs were recorded before, $20 \mathrm{~min}$ after, and $40 \mathrm{~min}$ after IIB injection. $A$, EPSPs were elicited by two action potentials and their peak amplitudes were measured. The paired-pulse depression ratio is plotted as a function of the intervals between the two stimuli $(n=6)$. $\boldsymbol{B}$, EPSPs were recorded at $1 \mathrm{~Hz}$. A train of action potentials $(900 \mathrm{at} 5 \mathrm{~Hz})$ was elicited in the presynaptic neuron from 0 to 3 min. Normalized and averaged EPSP amplitudes are shown $(n=5)$. C, The values shown in $B$ from 3 to 3.25 min are replotted on an expanded timescale with SEM. Noise levels of the amplitude 2.7-2.98 min were averaged and subtracted from the value after $3 \mathrm{~min}$. An exponential growth curve (blue, before; cyan, IIB $20 \mathrm{~min}$ ) representing the recovery rate of the EPSP amplitude. $p<$ 0.0001 , before vs IIB 20 min and $40 \mathrm{~min}$, one-way ANOVA for the curves; $p<0.01, p<0.001$, before vs IIB $20 \mathrm{~min}$ and vs 40 min Bonferroni's multicomparison test. $D$, EPSPs recorded at $1 \mathrm{~Hz}$. At time $=0,0.5 \mathrm{~m}$ sucrose was puff applied to the synapse for $20 \mathrm{~s}$. Normalized and averaged integral values for EPSPs are plotted against the recording time $(n=4)$. $\boldsymbol{E}$, The values shown in $\boldsymbol{D}$ from 20 to 35 s are replotted on an expanded timescale. An exponential growth curve representing the recovery rate of the EPSP integral is fitted to the values, and indicates that the time constant for EPSP recovery is significantly prolonged in the presence of myosin IIB fragments. $p<0.0001$, paired $t$ test for the curves.

ms after an AP; Fig. $6 B)$ is a bit slower than that of dynamin-2 and dynamin-3 (20 ms after an AP; Tanifuji et al., 2013), but both are in a similar timescale range, suggesting the possible role of myo$\sin$ VI for active zone clearance following the dynamin action in SV membrane trafficking.

The number (Zhu et al., 2009) and the position (Park et al., 2012) of exocytosis events affect RRP recovery rates. In addition, studies at the calyx of Held (Neher, 2010) indicate that rapid endocytosis is the rate-limiting step for RRP replenishment, suggesting that myosin VI, in addition to dynamin isoforms, may be crucial regulatory control factors for the membrane's response to cell activity. Indeed, in our current study, myosin VI knockdown impaired the ability of cells to translate high-frequency AP firing into recovery and maintenance of the RRP (Fig. 5).

\section{How is endocytosis involved in myosin-mediated SV resupply?}

Three isoforms of dynamin, an essential endocytic protein, select appropriate vesicle reuse pathways associated with specific neuronal firing patterns in SCG neurons (Tanifuji et al., 2013). Dynamin- $1,-2$, and -3 work individually to match vesicle reuse pathways, having distinct rate and time constants with physiological AP frequencies. Dynamin-3 resupplies the RRP with slow kinetics independently of the AP frequency but acted quickly, within $20 \mathrm{~ms}$ of the incoming AP. Under high-frequency firing, dynamin-1 regulates recycling to the RRP with fast kinetics in a slower time window $>50$ ms. Dynamin-2 displays a hybrid response between the other isoforms. In the present study, we ap- plied the same protocol to monitor the rate and the time constant for myosin-mediated RRP resupply. Myosin VI resupplied the RRP with slow kinetics but acted quickly, within $50 \mathrm{~ms}$ of the incoming AP, while myosin IIB resupplied the RRP with fast kinetics in a slower time window of $200 \mathrm{~ms}$ under high-frequency AP firing (Figs. 5-7). These results suggest that high-frequency firing activates dynamin-1, dynamin-2, and myosin IIB, while dynamin-2, dynamin-3, and myosin VI are activated independently of the AP frequency.

Furthermore, we applied temporal and spatial analysis for myosin IIB and myosin VI actions in relation to two distinct dynamin-1-mediated and dynamin-3-mediated endocytosis, using double $\mathrm{KD}$ of myosin IIB or myosin VI and dynamin-1 or dynamin-3 (Fig. $7 H-M$ ). Double KD of myosin IIB and dynamin-1 additively reduced EPSP recovery but did not affect the recovery rate with IIB-KD, suggesting that myosin IIB transports reserved SVs formed via the dynamin-1-mediated endocytosis. Additive effects of double KD suggest that the myosin IIB does not transport SVs formed via the dynamin-3-mediated endocytosis and that myosin VI does not transport SVs formed via dynamin-1-mediated endocytosis. On the other hand, similar levels of myosin VI and dynamin-3 double KD effect the VI-KD effect, suggesting that myosin VI transports SVs formed by dynamin-3-mediated endocytosis. Thus, in SCG neurons, highfrequency firing activates the endocytosis mediated by dynamin- 1 and dynamin-2, and the myosin IIB-mediated vesicle resupply to the RRP. In contrast, the endocytosis via dynamin-2 and dynamin-3, and the following SV transport by myosin VI to fill a shared RRP are activated independently of the AP frequency. 
$\mathrm{Ai}$

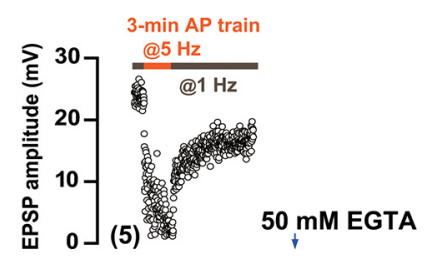

Bi

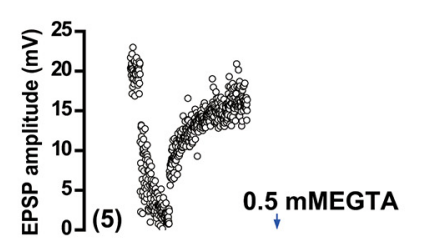

C i

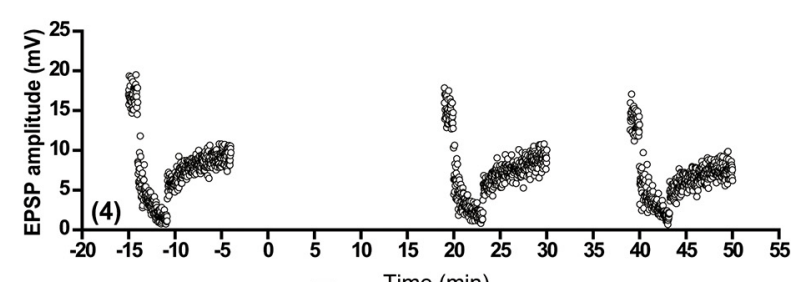

D
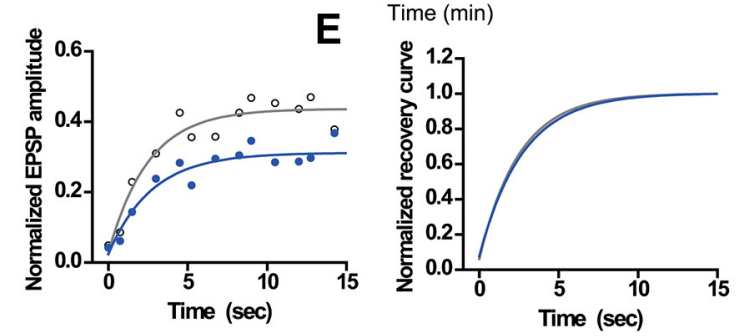
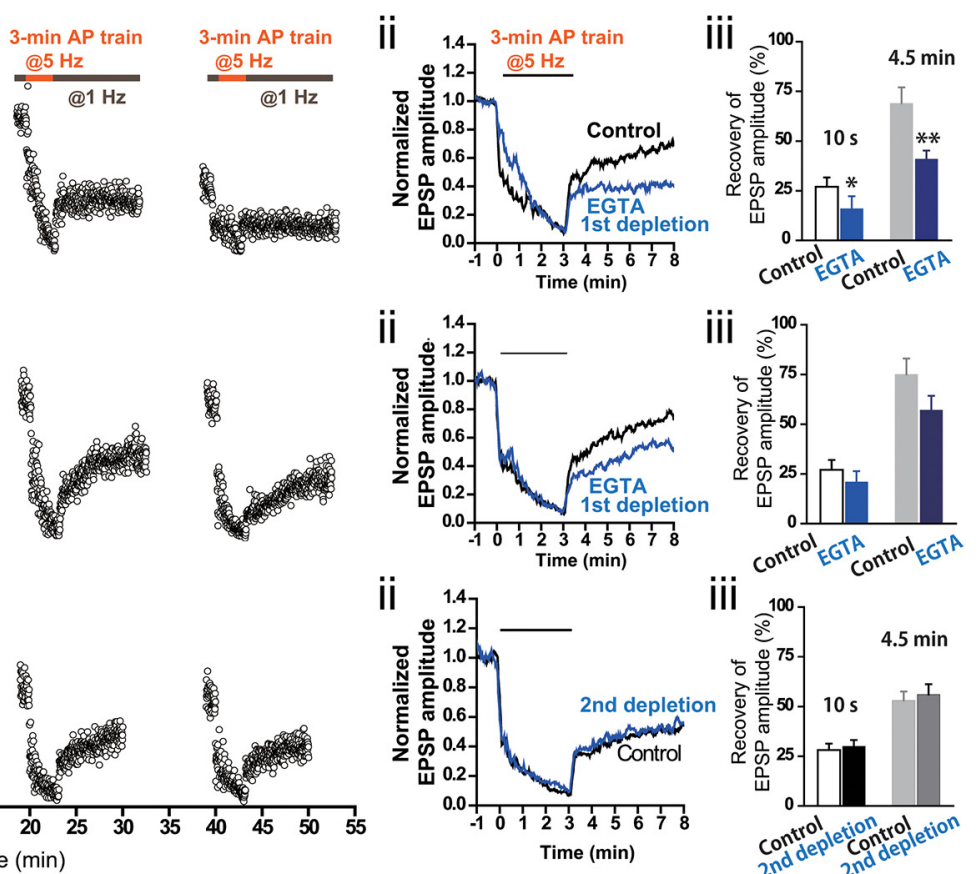

iii
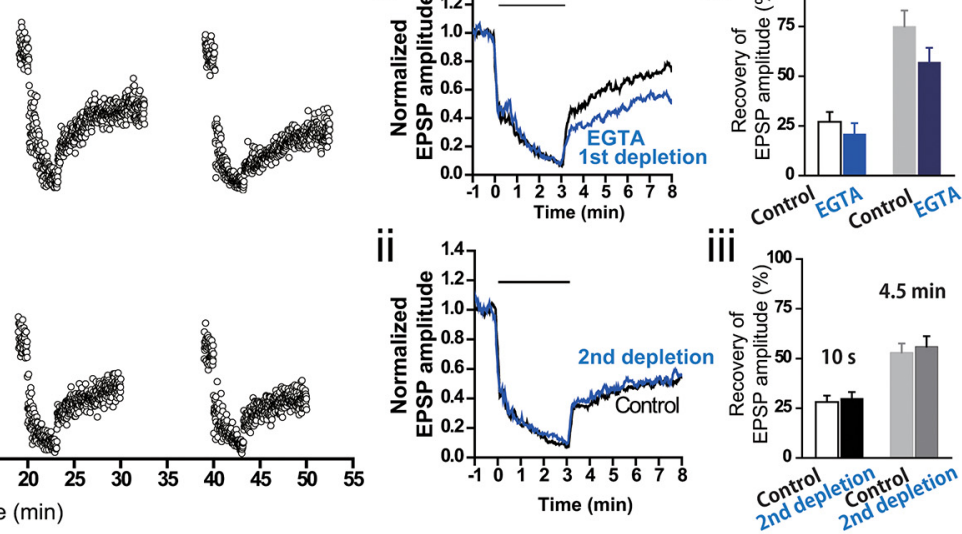

Figure 9. EPSP recovery after depletion of synaptic vesicle pools by train stimulation is $\mathrm{Ca}^{2+}$ dependent. EPSPs were recorded at $1 \mathrm{~Hz}$. EGTA was injected into presynaptic neurons at time $=0$ for $5 \mathrm{~min}$. The concentration in the injection pipette was $50 \mathrm{~mm}$ for $\boldsymbol{A}$ and $0.5 \mathrm{~mm}$ for $\boldsymbol{B}$. A train of action potentials ( $900 \mathrm{at} 5 \mathrm{~Hz}$ indicated as horizontal bars) was elicited in the presynaptic neuron 15-12 min before injection of EGTA, $20-23$ min after injection, and $40-43 \mathrm{~min}$ after injection ( $n=5$ for $\boldsymbol{A}$ and $\boldsymbol{B}$ ). As a control, EPSP amplitudes obtained from noninjected synapses are shown in $\mathbf{C}(n=4)$. $\mathbf{A i}$, Bi, and $\mathbf{C i}$, Averaged EPSP amplitudes are plotted against recording time. Aii, Bii, and $\mathbf{C i i}$, EPSP amplitudes shown in $\mathbf{A i}$, Bi, and $\mathbf{C i}$ are normalized and smoothed with a moving average algorithm and plotted with a line. Time $=0$ indicates the start of train stimulation at 15 min before EGTA injection (black line) and 20 min after injection (blue line). Aiii, Biii, and Ciii, Bar graph illustrating the recovery of EPSP amplitudes at $10 \mathrm{~s}$ (left two columns) and $4.5 \mathrm{~min}$ (right two columns) after cessation of the train stimulation. White and light gray columns: before EGTA injection. Blue and navy columns: at 20 min after injection. Black and dark gray: after the second deletion. Aiii, Statistically significant from control at ${ }^{*} p<0.05$ and ${ }^{* *} p<0.01$ (before vs at 20 min after injection) with paired $t$ test. Biii and (iii, $p=0.15$ at 10 s and 0.11 at $4.5 \min$ for $\boldsymbol{B}, p=0.35$ at 10 s and $p=0.42$ at $4.5 \mathrm{~min}$ for $\boldsymbol{C}$, before versus at 20 min after injection. $\boldsymbol{D}, \boldsymbol{E}$, Exponential growth curves fitted with averaged EPSP amplitudes shown in $A$ and the normalized curves in $E$. These results suggest that slow $\mathrm{Ca}^{2+}$ kinetics of $\mathrm{Ca}^{2+}$ signal does not contribute in the fast recovery of the RRP but contributes in the slow phase. These results are consistent with the effects of extracellularly applied Ca ${ }^{2+}$ chelators (Mori et al., 2014).

\section{The kinetics for the RRP replenishment}

During and after high-frequency firing, myosin IIB activation was accelerated, while myosin VI was activated independently of firing frequency (Fig. 5). After the RRP depletion with highfrequency firing, IIB-mediated recovery with fast kinetics $(\tau \leq$ 7.5 s), while VI mediated recovery with slow kinetics $(\tau \leq 3.6$ min) (Fig. 7, Control). The fast process corresponds to rapid RRP refilling with SVs from the reserve pool (RP) in an amphiphysin-dependent and dynamin-1-mediated and dynamin2-mediated endocytic pathway, while the slow process involves a gradual refilling of the RRP with SVs through an amphiphysinindependent and dynamin-2-mediated and dynamin-3mediated endocytic pathway that bypasses the RP (Lu et al., 2009; Tanifuji et al., 2013). Loss of function of myosin IIB slowed the RRP recovery through the dynamin-1 (and dynamin-2-)mediated endocytic pathway (Fig. 7), suggesting that IIB controls the kinetics and the size of the RP. During and after highfrequency firing a reduction in the RRP size accelerates the dynamin-1-mediated and dynamin-2-mediated endocytic pathway to fill the RP (Tanifuji et al., 2013), resulting in an increase in the RRP size cooperating with myosin IIB (Fig. 5C). Our results suggest that the overall kinetics of recovery do not change as a function of the size of the vesicle pool per se, but rather due to the pattern of AP firing that drives the recovery process. Thus, it is critical to have two pathways that can each respond to firing variation patterns to replenish the RRP.

\section{Vesicle pool replenishment is $\mathrm{Ca}^{2+}$ dependent}

How are myosin IIB and VI selectively activated by AP firing? For myosin IIB to be enzymatically active, its regulatory light chain subunit must be phosphorylated by a $\mathrm{Ca}^{2+} /$ calmodulindependent myosin light chain kinase. Furthermore, $\mathrm{Ca}^{2+} / \mathrm{cal}-$ modulin light chain binding activates myosin VI. Therefore, the putative step(s) requiring myosin function should also be $\mathrm{Ca}^{2+}$ dependent and sensitive to experimental calcium buffering. Introducing the slow $\mathrm{Ca}^{2+}$ buffer EGTA into presynaptic nerve terminals severely inhibited the slow phase of the RRP recovery and reduced the RP size after SV depletion (Fig. 9), while the fast $\mathrm{Ca}^{2+}$ buffer BAPTA-AM bath application slowed the fast phase of the RRP recovery (Mori et al., 2014). These results suggest that 
temporal and spatial transient increases in $\mathrm{Ca}^{2+}$ regulate activation of myosins for the RRP resupply. The requirement of myosin IIB in SV resupply monitored by evoked EPSP recovery, after $\mathrm{Ca}^{2+}$-dependent and $\mathrm{Ca}^{2+}$-independent depletion of the RRP with AP train and sucrose (Figs. 7, 8), suggests that myosin IIB is selectively activated by repetitive AP firing (Figs. $2 I, J, 5 A$ ), for the fast RRP replenishment with SVs from the RP (Figs. 7A, $8 D$ ). Whether there is a spatial synaptic organization to myosin isoform-dependent resupply pathways remains a challenging future issue.

\section{Conclusion}

Collectively, our findings provide a foundation for understanding the molecular mechanisms responsible for the selection and regulation of distinct SV trafficking complexes by electrical activity. We propose the novel idea that distinct myosin isoforms controlling unique vesicle pools may allow synapses to respond dynamically to rapid or complex AP bursts into EPSPs that reflect a cell's history of synaptic firing, while simultaneously maintaining the general capability for fast and stable SV resupply and maintenance after sparse single APs.

\section{References}

Baba T, Sakisaka T, Mochida S, Takai Y (2005) PKA-catalyzed phosphorylation of tomosyn and its implication in $\mathrm{Ca}^{2+}$-dependent exocytosis of neurotransmitter. J Cell Biol 170:1113-1125. CrossRef Medline

Chandrasekar I, Goeckeler ZM, Turney SG, Wang P, Wysolmerski RB, Adelstein RS, Bridgman PC (2014) Nonmuscle myosin II is a critical regulator of clathrin-mediated endocytosis. Traffic 15:418-432. CrossRef Medline

Cingolani LA, Goda Y (2008) Actin in action: the interplay between the actin cytoskeleton and synaptic efficacy. Nat Rev Neurosci 9:344-356. CrossRef Medline

Frost NA, Kerr JM, Lu HE, Blanpied TA (2010) A network of networks: cytoskeletal control of compartmentalized function within dendritic spines. Curr Opin Neurobiol 20:578-587. CrossRef Medline

Hammer JA 3rd, Sellers JR (2012) Walking to work: roles for class V myosins as cargo transporters. Nat Rev Mol Cell Biol 13:13-26. CrossRef Medline

Hosoi N, Holt M, Sakaba T (2009) Calcium dependence of exo- and endocytotic coupling at a glutamatergic synapse. Neuron 63:216-229. CrossRef Medline

Hotulainen P, Hoogenraad CC (2010) Actin in dendritic spines: connecting dynamics to function. J Cell Biol 189:619-629. CrossRef Medline

Inchauspe CG, Forsythe ID, Uchitel OD (2007) Changes in synaptic transmission properties due to the expression of $\mathrm{N}$-type calcium channels at the calyx of Held synapse of mice lacking P/Q-type calcium channels. J Physiol 584:835-851. CrossRef Medline

Kawasaki F, Hazen M, Ordway RW (2000) Fast synaptic fatigue in shibire mutants reveals a rapid requirement for dynamin in synaptic vesicle membrane trafficking. Nat Neurosci 3:859-860. CrossRef Medline

Kisiel M, Majumdar D, Campbell S, Stewart BA (2011) Myosin VI contributes to synaptic transmission and development at the Drosophila neuromuscular junction. BMC Neurosci 12:65. CrossRef Medline

Kisiel M, McKenzie K, Stewart B (2014) Localization and mobility of synaptic vesicles in myosin VI mutants of Drosophila. PLoS One 9:e102988. CrossRef Medline

Kneussel M, Wagner W (2013) Myosin motors at neuronal synapses: drivers of membrane transport and actin dynamics. Nat Rev Neurosci 14:233247. CrossRef Medline

Korobova F, Svitkina T (2010) Molecular architecture of synaptic actin cytoskeleton in hippocampal neurons reveals a mechanism of dendritic spine morphogenesis. Mol Biol Cell 21:165-176. CrossRef Medline

Krapivinsky G, Mochida S, Krapivinsky L, Cibulsky SM, Clapham DE (2006) The TRPM7 ion channel functions in cholinergic synaptic vesicles and affects transmitter release. Neuron 52:485-496. CrossRef Medline

Lee JS, Ho WK, Lee SH (2010) Post-tetanic increase in the fast-releasing synaptic vesicle pool at the expense of the slowly releasing pool. J Gen Physiol 136:259-272. CrossRef Medline
Lu W, Ma H, Sheng ZH, Mochida S (2009) Dynamin and activity regulate synaptic vesicle recycling in sympathetic neurons. J Biol Chem 284:1930 1937. CrossRef Medline

Ma H, Mochida S (2007) A cholinergic model synapse to elucidate protein function at presynaptic terminals. Neurosci Res 57:491-498. CrossRef Medline

Ma H, Cai Q, Lu W, Sheng ZH, Mochida S (2009) KIF5B motor adaptor syntabulin maintains synaptic transmission in sympathetic neurons. J Neurosci 29:13019-13029. CrossRef Medline

Magupalli VG, Mochida S, Yan J, Jiang X, Westenbroek RE, Nairn AC, Scheuer T, Catterall WA (2013) $\mathrm{Ca}^{2+}$-independent activation of $\mathrm{Ca}^{2+} /$ calmodulin-dependent protein kinase II bound to the C-terminal domain of CaV2.1 calcium channels. J Biol Chem 288:4637-4648. CrossRef Medline

Mochida S (1995) Role of myosin in neurotransmitter release: functional studies at synapses formed in culture. J Physiol Paris 89:83-94. CrossRef Medline

Mochida S, Kobayashi H, Matsuda Y, Yuda Y, Muramoto K, Nonomura Y (1994a) Myosin II is involved in transmitter release at synapses formed between rat sympathetic neurons in culture. Neuron 13:1131-1142. CrossRef Medline

Mochida S, Nonomura Y, Kobayashi H (1994b) Analysis of the mechanism for acetylcholine release at the synapse formed between rat sympathetic neurons in culture. Microsc Res Tech 29:94-102. CrossRef Medline

Mochida S, Few AP, Scheuer T, Catterall WA (2008) Regulation of presynaptic $\mathrm{ca}(\mathrm{v}) 2.1$ channels by $\mathrm{ca}(2+)$ sensor proteins mediates short-term synaptic plasticity. Neuron 57:210-216. CrossRef Medline

Mori M, Tanifuji S, Mochida S (2014) Kinetic organization of $\mathrm{Ca}^{2+}$ signals that regulate synaptic release efficacy in sympathetic neurons. Mol Pharmacol 86:297-305. CrossRef Medline

Murakami N, Chauhan VP, Elzinga M (1998) Two nonmuscle myosin II heavy chain isoforms expressed in rabbit brains: filament forming properties, the effects of phosphorylation by protein kinase $\mathrm{C}$ and casein kinase II, and location of the phosphorylation sites. Biochemistry 37:1989-2003. CrossRef Medline

Murakami N, Kotula L, Hwang YW (2000) Two distinct mechanisms for regulation of nonmuscle myosin assembly via the heavy chain: phosphorylation for MIIB and mts 1 binding for MIIA. Biochemistry 39:1144111451. CrossRef Medline

Nash JE, Appleby VJ, Corrêa SA, Wu H, Fitzjohn SM, Garner CC, Collingridge GL, Molnár E (2010) Disruption of the interaction between myosin VI and SAP97 is associated with a reduction in the number of AMPARs at hippocampal synapses. J Neurochem 112:677-690. CrossRef Medline

Neher E (2010) What is rate-limiting during sustained synaptic activity: vesicle supply or the availability of release sites. Front Synaptic Neurosci 2:144. CrossRef Medline

Neher E, Sakaba T (2008) Multiple roles of calcium ions in the regulation of neurotransmitter release. Neuron 59:861-872. CrossRef Medline

Osterweil E, Wells DG, Mooseker MS (2005) A role for myosin VI in postsynaptic structure and glutamate receptor endocytosis. J Cell Biol 168: 329-338. CrossRef Medline

Park H, Li Y, Tsien RW (2012) Influence of synaptic vesicle position on release probability and exocytotic fusion mode. Science 335:1362-1366. CrossRef Medline

Peng A, Rotman Z, Deng PY, Klyachko VA (2012) Differential motion dynamics of synaptic vesicles undergoing spontaneous and activity-evoked endocytosis. Neuron 73:1108-1115. CrossRef Medline

Rex CS, Gavin CF, Rubio MD, Kramar EA, Chen LY, Jia Y, Huganir RL, Muzyczka N, Gall CM, Miller CA, Lynch G, Rumbaugh G (2010) Myosin IIb regulates actin dynamics during synaptic plasticity and memory formation. Neuron 67:603-617. CrossRef Medline

Rizzoli SO, Betz WJ (2005) Synaptic vesicle pools. Nat Rev Neurosci 6:57-69. CrossRef Medline

Schneggenburger R, Meyer AC, Neher E (1999) Released fraction and total size of a pool of immediately available transmitter quanta at a calyx synapse. Neuron 23:399-409. CrossRef Medline

Seabrooke S, Stewart BA (2011) Synaptic transmission and plasticity are modulated by nonmuscle myosin II at the neuromuscular junction of Drosophila. J Neurophysiol 105:1966-1976. CrossRef Medline

Seabrooke S, Qiu X, Stewart BA (2010) Nonmuscle Myosin II helps regulate 
synaptic vesicle mobility at the Drosophila neuromuscular junction. BMC Neurosci 11:37. CrossRef Medline

Su Q, Cai Q, Gerwin C, Smith CL, Sheng ZH (2004) Syntabulin is a microtubule-associated protein implicated in syntaxin transport in neurons. Nat Cell Biol 6:941-953. CrossRef Medline

Sweeney HL, Houdusse A (2010) Myosin VI rewrites the rules for myosin motors. Cell 141:573-582. CrossRef Medline

Takagishi Y, Futaki S, Itoh K, Espreafico EM, Murakami N, Murata Y, Mochida S (2005) Localization of myosin II and V isoforms in cultured rat sympathetic neurones and their potential involvement in presynaptic function. J Physiol 569:195-208. CrossRef Medline

Tanifuji S, Funakoshi-Tago M, Ueda F, Kasahara T, Mochida S (2013) Dynamin isoforms decode action potential firing for synaptic vesicle recycling. J Biol Chem 288:19050-19059. CrossRef Medline

tom Dieck S, Sanmartí-Vila L, Langnaese K, Richter K, Kindler S, Soyke A, Wex H, Smalla KH, Kämpf U, Fränzer JT, Stumm M, Garner CC, Gundelfinger ED (1998) Bassoon, a novel zinc-finger CAG/glutaminerepeat protein selectively localized at the active zone of presynaptic nerve terminals. J Cell Biol 142:499-509. CrossRef Medline

Vicente-Manzanares M, Ma X, Adelstein RS, Horwitz AR (2009) Nonmuscle myosin II takes centre stage in cell adhesion and migration. Nat Rev Mol Cell Biol 10:778-790. CrossRef Medline

Vogl C, Tanifuji S, Danis B, Daniels V, Foerch P, Wolff C, Whalley BJ, Mochida S, Stephens GJ (2015) Synaptic vesicle glycoprotein 2A modu- lates vesicular release and calcium channel function at peripheral sympathetic synapses. Eur J Neurosci 41:398-409. CrossRef Medline

Wagner W, Brenowitz SD, Hammer JA 3rd (2011) Myosin-Va transports the endoplasmic reticulum into the dendritic spines of Purkinje neurons. Nat Cell Biol 13:40-48. CrossRef Medline

Watanabe S, Rost BR, Camacho-Pérez M, Davis MW, Söhl-Kielczynski B, Rosenmund C, Jorgensen EM (2013) Ultrafast endocytosis at mouse hippocampal synapses. Nature 504:242-247. CrossRef Medline

Watanabe S, Trimbuch T, Camacho-Pérez M, Rost BR, Brokowski B, SöhlKielczynski B, Felies A, Davis MW, Rosenmund C, Jorgensen EM (2014) Clathrin regenerates synaptic vesicles from endosomes. Nature 515:228233. CrossRef Medline

Wiedenmann B, Franke WW (1985) Identification and localization of synaptophysin, an integral membrane glycoprotein of Mr 38,000 characteristic of presynaptic vesicles. Cell 41:1017-1028. CrossRef Medline

Wu LG (2004) Kinetic regulation of vesicle endocytosis at synapses. Trends Neurosci 27:548-554. CrossRef Medline

Yano H, Ninan I, Zhang H, Milner TA, Arancio O, Chao MV (2006) BDNFmediated neurotransmission relies upon a myosin VI motor complex. Nat Neurosci 9:1009-1018. CrossRef Medline

Zhu Y, Xu J, Heinemann SF (2009) Two pathways of synaptic vesicle retrieval revealed by single-vesicle imaging. Neuron 61:397-411. CrossRef Medline 